$19^{\text {th }}$ Conference of the Asian Pacific Association for the Study of the Liver

\title{
Invited Abstracts
}


Presidential Lecutre

14 Feb, 2009 (Saturday), 11:00 - 11:30

Hall 5A

Treatment of Chronic Hepatitis B Infection - Year 2009 and Beyond

George KK Lau

Department of Medicine, The University of Hong Kong

Worldwide, chronic hepatitis B virus infection is a major cause of end-stage liver disease and hepatocellular carcinoma. While the past two decades have brought major advances in the availability of treatments to help delay or prevent these outcomes, treatment of chronic hepatitis B remains a serious challenge. With the recent availability of potent new nucleot(s)ide such as entecavir, tenofovir, telbuvidine and pegylated interferon (PEG-IFN)- $\alpha$ for the treatment of chronic hepatitis B infection, there is still a long way to go before we can have a more ideal form of management for this potentially dreadful disease. We will need better therapy which will enable us to induce lasting off-treatment sustained disease remission. From the natural history point of view, this will mean sustained full hepatitis B e-antigen seroconversion (loss of e-antigen, development of hepatitis B e antigen and reduction of serum HBV DNA $\leq 10^{4}$ ) or even hepatitis B surface antigen seroconversion. Being able to do this will allow us to shorten the duration of therapy and hence to avoid the cost, side effect and the risk of development of viral resistance with the use of prolonged nucleos(t)ide therapy. In order to facilitate better monitoring of anti-hepatitis B therapy, the development of standardized assay for serum viral antigen quantitation will be of great value. This is exemplified by the lack of strong relationship between the magnitude of serum HBV DNA suppression and the rate of hepatitis B e antigen and surface antigen seroconversion, especially when one is using nucleos(t)ide therapy. In the very near future, based on advanced technology platform available for more detailed genetic, immunological and virological studies, I believe that we will be able to further optimize hepatitis B therapy in a personalized fashion. On the other hand, with more data generated from detailed immunological and virological kinetics study, a more rational basis for combination therapy will be developed so that more effective and less costly therapy will be made available to our patients. Last, but not least, I urge for concerted effort with various disciplines in Asia-pacific region, to deal with hepatitis B infection and I believe that one day, we will have a more perfect solution.

\section{Kunio Okuda Lecture 14 Feb, 2009 (Saturday), 11:30 - 12:00} Hall $5 \mathrm{~A}$

\section{Liver Transplantation for Hepatocellular Carcinoma \\ Sheung-Tat Fan}

Department of Surgery, The University of Hong Kong

The Milan criteria are commonly used to determine the suitability of Child $\mathrm{B} / \mathrm{C}$ hepatocellular carcinoma (HCC) patients for undergoing liver transplantation, but the accuracy is controversial. To improve the current liver allocation system, it is important to study the biological behavior of HCC.

Among the different possible ways to predict the tumor biological behavior, studies have shown that the response of a tumor to non-surgical treatments, such as transarterial chemoembolization, and tumor differentiation grade are not totally predictive of the biological behavior of HCC.

Numerous studies have been carried out on the prognostic values of genes/markers in relation to various aspects of hepatocarcinogenesis. AA454543 and extracellular matrix metalloproteinase inducer, CD147, for example, have been shown to function as cell invasion and are predictive of tumor recurrence after liver transplantation. Because not more than $70 \%$ of the tumors express the same gene, more than one gene is needed to accurately predict the outcome of treatment. However, no such study has been proved clinically applicable.

In obtaining tumor tissues for genetic tests, tumor biopsy inevitably poses a risk of cancer cell dissemination into the circulation or cancer cell spillage into the peritoneal cavity. An alternative way is to examine the circulating cancer cells. To detect the circulating cancer cells, we may employ immunocytochemistry to identify the surface marker of the cancer cell, identify the protein secreted by the cells using EPISPOT assay, or measure the quantity of free nucleic acid released from mRNA of the cancer cells. Alpha-fetoprotein and plasma albumin mRNA are possible markers of tumor cells but large scale clinical studies have not been performed to validate their usefulness.

The cancer stem cell is a more recent concept. It is hypothesized that it has the ability to deposit and grow in the distant organs and liver graft to produce recurrence. The cancer stem cell of $\mathrm{HCC}$, identified by $\mathrm{CD} 45^{-}, \mathrm{CD} 90^{+}$and $\mathrm{CD} 44^{+}$, have recently been found not only in tumor tissues, but also in the blood in $90 \%$ of HCC patients, whereas in the blood of normal persons and cirrhotic patients, they are totally absent. It was found that the number of circulating cancer stem cells can predict both intrahepatic and extrahepatic tumor recurrence. The prediction ability appears to be higher than that of plasma albumin mRNA. CD44 antibody has also been shown to be useful for prevention of HCC recurrence after liver transplantation in animal models. In conclusion, blood is the preferred or clinically applicable sample source of study of HCC biological behavior. The focus of research is on the circulating cancer cell marker. On this aspect, the cancer stem cell may be the focus of future research because eradication of the circulating cancer stem cell may render a patient tumor-free after transplantation. In the future, the molecular screening may play an important role of selecting the appropriate patients to receive liver transplantation. Studies on such biomarkers will also provide opportunities of designing target agents to regress the tumor before transplant or prevent it from recurring after transplantation.

\section{State-of-the-Art Lecture \\ 14 Feb, 2009 (Saturday), 14:00 - 14:30}

Hall $5 \mathrm{~A}$

Hepatitis B Virus Infection and Hepatocellular Carcinoma: The Past and Future Prospectives

Masao Omata

University of Tokyo

The major discovery in the field of "hepatology" is obviously the identification of Australia Antigen in 1964 by Dr. Blumberg, Nobel Prize laureate. In addition, Dr. Beasley's epidemiological survey on 27,000 individuals clearly demonstrated the relation of this interferon and HCC. The universal vaccination will eventually reduce the infection, and, thus, occurrence of HCC in Asia. Thus, it remains the need of effective treatment for patients who already have been infected and diseases of varying severity. The discovery of reverse transcriptional process by J Summer and B Mason lead the development of reverse transcriptional inhibitors.

In my talk, I would like to address the past and the future perspective on the theme of HBV and HCC.

State-of-the-Art Lecture 15 Feb, 2009 (Sunday), 10:00 - 10:30

Hall 5A

Acute Liver Failure

Roger S. Williams

Institute of Hepatology, UCL Medical School, London

The three important phases, well accepted in the management schedule, are as follows:

1) Early identification of causes requiring specific treatment such as hepatic lymphoma, the Budd-Chiari Syndrome, ischaemic hepatic necrosis, fulminating septicaemia, Wilson's Disease and reactivation of HBV in chronic carriers.

2) Institution of early monitoring and optimal intensive care for multi-organ involvement to improve chances of spontaneous recovery 
or of transplantation. Deteriorating encephalopathy with cerebral oedema is related to systemic inflammatory response and infections need to be treated aggressively along with maintenance of the circulation and support of the organ function by enhancing tissue perfusion through the administration of NAC. The value of hypothermia in lowering intracranial pressure has yet to be proven and is dependent on ICP monitoring by Bolt insertion which in recent studies has failed to show survival advantage.

3) Assessment of the need for transplantation based on strong positive predictive values provided by the King's or Clichy Criteria. A significant percentage $(50 \%-60 \%)$ of those not fulfilling criteria also progress. Even with priority allocation systems, a Cadaver organ may not be obtained in time and experience to-date with Living Donor Liver Transplantation is encouraging. The MARS artificial support device has corrective effects on the disturbed pathophysiology of ALF and may be used as a bridge to transplant. It might also be expected to enhance the rate of spontaneous recovery through better control of circulatory changes including those affecting the brain. Challenges to future research include the significant number of cases where no aetiology is identifiable - a category which accounts for $15-30 \%$ of cases in the West. An answer to whether these are due to idiosyncratic reactions to drugs and substances not identified, or to auto-immune disease, or to unrecognised acetaminophen hepatotoxicity as shown by findings of a significant percentage with acetaminophen - protein adducts in the serum, could point to different approaches in management.

\section{State-of-the-Art Lecture 15 Feb, 2009 (Sunday), 10:30 - 11:00 Hall 5A}

The Insight into the Human Liver Proteome

Fuchu He

State Key Laboratory of Proteomics, Beijing Proteome Research Center, Beijing Institute of Radiation Medicine

The Human Liver Proteome Project (HLPP) is a large-scale international collaborative initiative now focusing on the proteomic analysis of the human liver in its different conditions. In the past five years, we investigated the proteomes of the Chinese fetal liver tissues, French adult liver tissues and Chinese adult liver tissues in turn.

Recently, after analyzing the proteome from the Chinese adult liver tissues, which comprised of 6,788 identified proteins in $95 \%$ confidence with at least two peptides matching, we turn to the proteomic analysis of the different organelles as well as the different kinds of liver cells. We succeeded in identifying 5882 proteins with 2 peptides or more in $95 \%$ confidence from plasma membrane, nucleus, cytoplasm, mitochondria, rough endoplasmic reticulum and smooth endoplasmic reticulum in human liver cells, which will be compared with those proteins identified from the human liver tissues. As well, 4969 proteins were identified from the same organelles of the mouse liver.

In order to effectively enrich the low-abundance proteins, especially those with modifications, we developed several new methods, which were obviously more useful, such as dephosphorylation of phosphopeptides by cerium oxide and specific capture of phosphopeptides on MALDI mass spectrometry. In addition, on the basis of our past results of the protein-protein interactions, a protein array of about 5000 unique liver ORFs was screened by yeast two hybrid mating method and 1632 protein-protein interactions were discovered. Of them, 1270 interactions were verified by yeast retransformation assay.
State-of-the-Art Lecture

16 Feb, 2009 (Monday), 10:30 - 11:00

Hall 5A

\section{Advances in Hepatitis C Infection}

Michael P. Manns

Medical School of Hannover, Germany

The treatment of hepatitis $\mathrm{C}$ is one of the most active fields in medicine. Untreated acute hepatitis $\mathrm{C}$ develops chronicity up to $80 \%$. Once acute hepatitis $\mathrm{C}$ infections become diagnosed, early treatment prevents chronicity and leads to a cure in more than $90 \%$ of the patients. Immediate treatment is pursued with conventional interferon (IFN) monotherapy or with pegylated interferon (PEG-IFN) for up to 24 weeks. Alternatively, a delayed treatment after 12 weeks is started with a combination of PEG-IFN plus ribavirin for 24 weeks in those patients who are still HCV RNA positive after 12 weeks. At present, the German Network of Competence "Hep-Net" is conducting a national multi-centre study to prepare immediate treatment with monotherapy versus delayed combination treatment of PEG-IFN plus ribavirin.

The standard of care for chronic hepatitis C since 2001 is the combination of PEG-IFN plus ribavirin. Genotype 1 is treated for 48 weeks, while genotypes 2 and 3 are treated for 24 weeks. In recent years, therapy for hepatitis $C$ has been optimized by individualizing of treatment according to genotype and early treatment response. For early treatment response, rapid virological response (RVR) has become very important. This means whether patients become HCV RNA negative in serum by week 4 . Patients with genotype 2 and 3 who achieve RVR can be treated for $12-16$ weeks. Genotype 1 patients with low pretreatment viral load (HCV RNA $<600000$ copies $/ \mathrm{ml}$ ) and RVR are treated for 24 weeks only. For all genotype 1 patients' early virological response (EVR), e.g. response after initial 12 weeks of treatment has become very important to guide treatment in genotype 1 patients. EVR is defined as $>2 \log 10$ decline in HCV RNA or negative HCV RNA by week 12 . If genotype 1 patients do not develop a decline in HCV RNA by more than 2 $\log$ treatment is stopped since success rates are minimal. If HCV RNA declines by $>2 \log 10$ and becomes negagtive by weeks 2472 week therapy is nowadays regarded as justified. In case HCV RNA is still detectable by week 24 therapy as to be terminated. The final goal of treatment is to achieve negative HCV RNA in serum 24 weeks after treatment, so called sustained virological response (SVR). Long term studies for all the available interferons have shown that relapse rates beyond 24 weeks are for the end of treatment are extremely rare. Virological response five years after the end of treatment is between $97 \%$ and $99 \%$. Thus chronic hepatitis $\mathrm{C}$ has become the first chronic viral infection that can be cured. At the present time, new interferons are under development as well as small direct antiviral monocules, in particular targeting the HCV NS3 protease or HCV 5B polymerase. These drugs are now in Phase I, II or early phase III of clinical development. However they have to be used together with both interferon and ribavirin. Most advanced in development are the protease inhibitors, Telaprevir and Boceprevir. They are very promising to reduce duration of treatment for genotype 1 treatment naïve patients from 48 to 24 weeks and at the same time to improve SVR rates to 55 to $65 \%$ in treatment naïve HCV genotype-1 patients. Preliminary results of phase 2 studies in non-responders or relapsers to previous standard of care (Peg-interferon plus ribavirin) have been published for telaprevir in combination with standard of care. First results indicate that $41 \%$ of non-respnders and $75 \%$ of relapsers achieve SVR after 24 weeks of therapy. Several HCV polymerase inhibitors are under development. HCV polymerase inhibitors like Valopicitabine and R1626 already underwent phase 2 studies but development had to be stopped due to safety concerns. Other like R 7126 are still under clinical development. These new small antiviral hepatitis $\mathrm{C}$ drugs are associated with viral resistance and new side-effects including itching, leucopenia, thrombocytopenia, anemia and gastrointestinal symptoms. However, the recent progress in the treatment of hepatitis $\mathrm{C}$ is a great success. Over recent years, the sustained virological response rates have been improved for genotype 2 and 3 patients to more than $80 \%$. Furthermore, acute hepatitis C can be cured in more than $90 \%$ with interferon monotherapy. Hepatitis C genotype 1 patients can be cured up to $60 \%$. Several new direct antiviral drugs like HCV protease and polymerase inhibitors are under clinical development and will reduce the duration of treatment in genotype 1 patients as well as improve the overall cure rates. Hopefully they also will help cure $\mathrm{HCV}$ in non-responders to previous Peg interferon plus ribavirin therapy. Hopefully liver transplantation will not be necessary for hepatitis $\mathrm{C}$ induced liver disease in the not too far distant future. 


\section{State-of-the-Art Lecture}

16 Feb, 2009 (Monday), 11:00 - 11:30

Hall 5A

The Lesson on HBV Virology from Experimental Models
Jörg Petersen

University Hospital Hamburg-Eppendorf, Germany
Editor's Forum - Expert's Tips on Publication

15 Feb, 2009 (Sunday), 10:30 - 12:00

Theatre 1

How to Prepare Your Manuscript to the Hepatology International, An
Official Journal of APASL
Masao Omata
University of Tokyo, Japan
In this session, I will discuss the followings;
1. How and when we created a new Journal, Hepatology International, for
the APASL?
2. What types of articles we are waiting for publication?
3. How to submit the Journal and what is Editorial Manager ${ }^{\mathrm{TM}}$ ?
4. Who are reviewing your manuscripts?
5. How soon the manuscripts will be reviewed?
6. Is Impact Factor given to the Journal?

State-of-the-Art Lecture

16 Feb, 2009 (Monday), 11:30 - 12:00

Hall $5 \mathrm{~A}$

Evolving Challenges in Hepatic Fibrosis

Scott L. Friedman

Mount Sinai School of Medicine

The understanding of hepatic fibrosis, or the liver's scarring response has emerged as a major focus of current research in hepatology. There are several challenges evolving in the field. These include: 1) We need better markers of fibrosis stage and disease activity; 2) Which patients with chronic liver disease are most likely to progress?; 3) What makes cirrhotics decompensate?; 4) We need a 'proof-of-concept' antifibrotic trial.

Hepatic stellate cells (HSC) are the primary source of extracellular matrix in normal and fibrotic liver. They undergo an "activation" or transdifferentiation in progressive stages yielding a cell type that is highly proliferative, fibrogenic and contractile. Activation of HSCs is the central event of fibrogenesis, combined with recruitment of other mesenchymal cells that also become activated myofibroblasts. Exciting progress has been made in understanding the molecular basis of this process. Major advances include: (a) elucidation of effects of key cytokines on and their signaling pathways in HSC; (b) understanding of transcriptional regulation of HSC activation; (c) characterization of matrix proteases and their inhibitors; (d) demonstration of apoptosis as an important event in resolution of hepatic fibrosis and identification of its mediators; (e) elucidation of the complex and dynamic interaction between HSC and matrix; (f) understanding of the role of other cellular elements in hepatic fibrosis and their interaction with HSC. In addition, clinical studies have begun to identify host genetic polymorphisms that may soon predict risk of fibrosis progression.

Several new areas of rapid progress in the past 5-10 years have also taken root, including: 1) identification of different fibrogenic populations apart from resident stellate cells, for example portal fibroblasts, fibrocytes and bone-marrow derived cells, as well as cells derived from epithelial mesenchymal transition; 2) emergence of stellate cells as finely-regulated determinants of hepatic inflammation and immunity; 3) elucidation of multiple pathways controlling gene expression during stellate cell activation including transcriptional, post-transcriptional and epigenetic; 4) recognition of disease-specific pathways of fibrogenesis; 5) re-emergence of hepatic macrophages as determinants of matrix degradation in fibrosis resolution and the importance of matrix cross linking and scar maturation in determining reversibility; 6) hints that hepatic stellate cells may contribute to hepatic stem cell behavior, cancer and regeneration. Clinical and translational implications of these advances have become clear, and have begun to impact significantly upon the management and outlook of patients with chronic liver disease.

The impact of cirrhosis on accelerating the risk of $\mathrm{HCC}$ is well established. At least six features of cirrhosis could predispose to HCC. These include: 1) Enhanced oxidative stress and DNA damage; 2) Matrix bound growth factors that promote survival and mitogenesis of hepatocytes; 3) Telomere shortening; 4) Enhanced inflammation associated with fibrosis; 5) Reduced NK cell mediated killing of tumor cells; 6) Activated stellate cells may promote stem cell expansion.

\section{Tips to Submit a Basic Research Paper}

Massimo Colombo

Fondazione IRCCS Maggiore Hospital, Mangiagalli and Regina Elena and University of Milan, Italy

Publishing research provides visibility, is evidence of productivity, and is necessary for getting funds. The experts agree: "Publish or Perish is still alive and well in the research community". Getting a paper accepted in a scientific journal, requires a lot of practice and many skills. In 1995, Byrne analyzed the reasons why most manuscripts submitted to medical journals are rejected for publication through a questionnaire-based survey. The Study involved a random sample of 50 peer reviewers for JAMA, 67 editors-in-chief of a sample of prominent journals and 25 Nobel laureates in physiology and medicine of the preceding decade. The compliance to the survey was poor since it gathered an overall response rate of $22 \%$ only. Study design problems $(71 \%)$ emerged as the most common cause of rejection, followed by inadequate details in Methods, conclusions unsupported by data and results marginal, predictable and trivial. The survey revealed that one possible reason for study-design flaws and inadequate details in the methods might be that, when reading a medical journal, many busy physicians skip the methods section and therefore give this section too little attention when they write their own papers. A manuscript needs to match the interests of the journal as closely as possible and the authors are encouraged to get familiar with publication standards, by carefully reading the journal's Information for Authors. It is also worth learning how the reviewers of the paper will think, and reviewing papers for journals might be very helpful to this end. Authors are encouraged not to give up, i.e. if the paper is rejected the authors should accept the Editor's and Reviewers' comments as a free expert consultation and revise the paper accordingly. The scientific review, indeed, is a very difficult process that takes into consideration a number of points beyond the formal critique transmitted to the authors. These include, among other things, the confidential comments to the Editors which address the importance of the scientific message as well as the originality and the overall design and evaluation of the paper together with well-constructed comments. The final editorial decision is a careful reply to the authors that put every piece together including priority of publication and suitability for the Journal. The authors should not forget that most papers rejected by top journals are published elsewhere. Science is a successful enterprise whose success depends crucially on the efficacy and honesty of its communicative practices.

Conclusions: To publish in top-grade peer-reviewed journals a clear scientific objective should be identified and solid data with impeccable methodology should be produced; further, it should not be forgotten that a well-written paper will convey a clear message. We should always remember that the publication system is based on trust and is largely a code of honour. 


\section{Proper Design and Conduct of Clinical Trials}

Patrick S. Kamath

Miles and Shirley Fiterman Center for Digestive Diseases

Mayo Clinic, Rochester, United States

New treatments in clinical medicine are most accepted if they are based on the results of a well-conducted randomized control trial (RCT). A well-conducted RCT has some requirements, the most important of which is that the study should aim to demonstrate relative differences between the new intervention and the standard treatment that are "meaningful". In addition, a RCT should not only address efficacy of a new treatment, but also safety. Therefore, not only should the studies be adequately powered to detect a statistically significant benefit, but the study should be for a long enough duration that side effects of the treatment will be apparent.

The duration of the clinical trial is critical. RCT's that are stopped early are, unfortunately, becoming increasingly more common. More importantly, such trials show implausibly large treatment effects since the number of "events" is small. Clinicians should, therefore, review the results of randomized control trials that are stopped early with considerable skepticism, especially when the new treatment is expensive. An argument often used in support of stopping a study early is that the larger patient community can benefit from the new treatment. In truth, this is not usually the case since dissemination of reports of the study is usually delayed. The reason for RCT's being truncated is that stopping a study early benefits several parties. Probably the most important issue is the ethics of treatment trials. These involve the role of "clinical equipoise", therapeutic misconceptions, non-financial conflict of interest, financial conflict of interest, as well as authorship. Clinical equipoise indicates genuine uncertainty among experts or the perceived risk/benefit of various treatments. That is, experts should determine that there is perceived equivalent risk/benefit ratio between different treatments. For example, in patients with hepatic encephalopathy, the role of lactulose treatment is in "equipoise". The clinician investigators' dilemma here is that the investigator in him wants evidence that lactulose is a better treatment than placebo, but the clinician puts the needs of the patients first. That is, should the clinician put a patient with hepatic encephalopathy on a placebo. The investigator's duty is to follow the protocol exactly, while the clinician's duty is to tailor the treatment to the best interest of the patient.

In placebo control trials, it would be ethical to add a standard drug or placebo to standard therapy. However, it would be unethical to use a study drug or placebo instead of standard treatment. Therefore, it is very important to agree upon what is standard treatment in particular situations. Often, the efficacy of such treatments is weak and there is lack of consensus in the overall medical community. This is where institution review boards have to balance the conflicting evidence with the opinion of local physicians managing similar patients.

Conflicts of interest are inevitable in academia, and the challenge is not to eradicate them but to recognize and manage them effectively. Conflicts of interest can be non-financial or financial. There are uniform requirements for manuscripts submitted to biomedical journals, and authors should have substantive intellectual contributions to a published study.

How to Write and Publish a Clinical Rsearch Paper

Samuel S. Lee

University of Calgary, Calgary, Canada

These suggestions are based on my $>20$ years experience as an author and manuscript reviewer, and also on several years work as a journal editor including being editor-in-chief of Liver International since 2007. Recommendations can be grouped into 4 areas of a clinical research project: 1) doing the project, 2) writing the paper, 3) submitting the paper, 4) after the first decision.

1. A) Before starting the project, ideally meet with all potential authors and agree on division of tasks and assignment of authorship including the order of authors. The criteria for authorship are written in several journals; 'gift authorship' which is notoriously common in Asia, is unethical and strongly discouraged by all journals. B) Agree on who will write the first draft or which sections. C) Keep notes so if there is any conflict or dispute later, the notes can be used. D) Most clinical studies require a reasonable knowledge of biostatistics. If nobody amongst the authors is expert in this area, consult a statistician.

2. Writing the paper. A) start writing it during the study (methods, introduction) and finish the draft soon after the study is finished. Procrastinating usually leads to inaccuracies and a delay may mean that somebody else 'wins the race'. B) Choose the simplest but most descriptive title. Avoid overly long titles. C) The purpose of an 'introduction' is to explain the rationale of why the study was done. Keep it simple and brief. D) Give enough details in the 'methods' to allow someone else to replicate your work if needed. Standard methods like PCR, etc need not be explained but can be supported by a reference. E) Show results simply and use figures to illustrate important or difficult points. Do not duplicate data in tables/text and figures. F) 'Discussion': discuss and place your findings in context of previous literature. Accentuate the positives and do not highlight the weaknesses of your study. Keep speculations to a minimum. Do not repeat statements already made in the introduction and results sections.

3. Submitting the paper: A) Choose the appropriate journal-look at an issue. Does it publish papers similar to yours? B) Read carefully the journal's 'instructions to authors' and adhere completely to these. C) Proofread the paper before submission. Use the 'spellcheck' and 'grammar-check' features of the word-processing programs to fix errors. D) Make sure the manuscript looks as clean and errorless as possible. All fonts in the text should be the same type and size. Figures should look easily interpretable with large lettering and symbols. They should not look too complicated or too 'busy'. If a reader must spend more than 30 seconds trying to understand your figure, they will probably not bother. If that reader is an editor or reviewer, they will be unimpressed. E) Most journals allow referee suggestions. Suggest persons who will give you a fair and hopefully favorable review. Avoid suggesting your friends, particularly from your own country. Editors are not stupid; you will have a better chance of having a reviewer suggestion selected if they are from other countries.

4. after the first decision. A) If rejected, don't appeal; almost all are unsuccessful. B) read the comments carefully and try to improve the paper before submitting it elsewhere. C) If allowed a revision, carefully address every comment by the editor and by the referees. I usually make all the minor changes they want. If a major change is not possible for you to make, or you disagree with the referee on a specific point and you feel it will significantly negatively impact your paper, defend your point of view vigorously, with supporting references from the literature or additional data or both. Note that fighting the journal on an important point especially when they have asked for additional data or studies, is usually fruitless. For example, if they want more data and you choose instead to contest the need for such data, most referees/editors reject such papers. 


\section{Concurrent Session 1: HCV Treatment 14 Feb, 2009 (Saturday), 15:30 - 17:00 Convention Hall A}

Imagining the Future: New Molecules for $\mathrm{HCV}$

John G. McHutchison

Duke Clinical Research Institute, Durham, United States

The advent of newer therapies for the treatment of patients with viral hepatitis will continue to create an enriched environment for clinical research. It behooves all involved in the development of these newer agents, irrespective of their biological targets, to determine the most efficient and effective use of these new regimens. The overall goal should not simply be " to get a drug approved" but rather how to navigate the regulatory hurdles as efficiently and safely as possible, while at the same time addressing a number of key issues.

These major issues involve, but are not limited to the following. Determining the most appropriate combination of new agents to maximize efficacy, while at the same time minimizing adverse events. These regimens may also differ according to the targeted populations (a likely scenario is that regimens for African Americans with hepatitis C, may vary compared to other, easier to treat populations with the same disease).

Evaluating each new regimen in terms of its "resistance" profile in combinations with other agents. Strategies and strict guidelines for evaluating resistance that will allow the safest regimens to be developed for patients in this respect will need to be developed and implemented across the board. Systems to evaluate whether certain classes of drugs interact more positively with others in terms of patient outcomes (for example, protease and polymerase inhibitors for $\mathrm{HCV}$ infection) in the clinic will be necessary, as will our ability to evaluate whether certain drugs have "class" effects will also be important. Strategies will be required to follow and treat patients who have developed treatment resistant mutations, but have failed to eradicate the viral infection in question.

Future strategies should clearly be aimed at eradicating infection (as is now possible for $\mathrm{HCV}$ infection, but not as yet, $\mathrm{HBV}$ infection), rather than developing expensive, long-term suppressive therapies whenever possible. Virology portals and similar systems with resistance testing functions made available to health care practitioners will also be of utmost importance to manage patients most effectively.

As newer therapies emerge and are developed and approved, up to date, effective guidelines and cost effectiveness analyses will need to be promptly developed. The final step should be then to implement quality outcome measures in the community, to assure that appropriate patients are treated and receiving the most appropriate therapies, in a quantifiable manner.

\section{The Difficult-to-Treat Patient with Chronic Hepatitis C Infection Robert G. Gish}

California Pacific Medical Center, San Francisco, United States

Hepatitis $\mathrm{C}$ is a worldwide infection that affects more than 170 million individuals. The lifetime risk of cirrhosis remains around $20 \%$ and approximately $20 \%$ of those patients who develop cirrhosis will develop liver cancer. The only management for hepatitis $\mathrm{C}$ patients with end-stage liver disease with liver failure with or without liver cancer remains liver transplantation. The ultimate goal of management of hepatitis $\mathrm{C}$ is to cure patients, preferably, before they develop cirrhosis or cancer and before they develop the need for liver transplantation. Unfortunately, there are a large number of patients who have failed interferon or who are not candidates for this complex treatment. The patients who are "difficult to treat include patients with the profile of a low response and low cure rate to interferon-based antiviral therapy include:

\section{Patients with HIV co- infection.}

2. Patients who have advanced fibrosis or cirrhosis.

3. Patients with fatty liver (NASH) disease, especially those patients with documented insulin resistance.

4. Patients with defective immune systems such as patients with organ transplants.
5. Specific ethnic groups including African Americans.

6. Patients with medical or psychological contraindications to interferon-based therapies who may need dose reductions during treatment.

7. Patients with contraindications to ribavirin-based therapy such as anemia or cardiovascular disease who may not be able to receive full dose ribavirin therapy.

8. Patients without adequate health care resources who may be noncompliant or non adherent to a therapeutic regimen.

The major focus of this discussion will be patients with advanced fibrosis and cirrhosis, and patients with fatty liver with insulin resistance as well as patients with ethnic backgrounds that have low response rates due to changes in intracellular responses to interferon and or ribavirin. The viral and immune mechanistic explanations for low cure rates in patients with cirrhosis or insulin-resistance have not been clearly defined. Immune responses, both intra- and extracellular, are probably modified in each of these important clinical conditions. Cirrhosis is a relative condition of immunosuppression. $\mathrm{T}$ cell dysfunction has been reported in this clinical setting as well. Insulin resistance appears to interfere with intracellular mechanisms to suppress or clear $\mathrm{HCV}$ infection. Attempts to override these inhibitory pathways have not yet been clearly or fully established; yet, patients with fatty liver who undergo weight loss appear to have a higher response rate to Interferon-based therapies. Changes in response to interferon that are based on genetic and ethnic backgrounds are being investigated and are not fully understood.

In closing, the difficult-to-treat patient with hepatitis $\mathrm{C}$ continues to be a dominant portion of hepatologists and other clinicians' practice profiles. Additional information is needed and additional therapeutic options are required to increase the cure rate in this patient population.

\section{Gene Therapy in Hepatitis C Virus Infection}

Lai Wei

Peking University People's Hospital, Peking University Hepatology Institute, Beijing, China

Chronic hepatitis $\mathrm{C}$ virus (HCV) infection can lead to cirrhosis and hepatocellular carcinoma. As a milestone, interferon alfa pegylation and combine with ribavirin improve sustain viral response for chronic hepatitis $\mathrm{C}$ treatment significantly. However, around half patients remain nonresponse or relapse due to different viral and host factors, therefore, requirement of alternative options for $\mathrm{HCV}$ infection is necessary. Progress in $\mathrm{HCV}$ molecular research and technologies make alternative therapy possible. The researches on $\mathrm{HCV}$ lifecycle show that $\mathrm{HCV}$ replication is dependent upon a few of viral and host cell factors. Internal ribosomal entry site (IRES) in the 5 ' non-translated region(NCR) of $\mathrm{HCV}$ plays an important role in ribosomal assembley and mediates translation of the HCV polyprotein. The viral envelope glycoproteins E1 and E2 are regarded as the critical determinants for virus cell entry. Non structure(NS) gene 3 to $5 \mathrm{~b}$ region is an essential component of the $\mathrm{HCV}$ replication complex. Therefore, they are regard as potential genetherapy targets for chronic hepatitis C. In addition to these viral elements, some host factors including geranylgeranylated protein FBL-2, the immunophilins cyclophilin B, FKBP8 are also regarded as potential targets for antiviral therapy. More recently, hnRNP D, interacts with the 5 ' border of HCV IRES (stem loop II), and promotes translation of HCV mRNA. Meanwhile, Human La protein has been implicated to facilitate internal ribosome entry site (IRES) mediated translation of hepatitis $\mathrm{C}$ virus $(\mathrm{HCV})$. More recently, a liver specific noncoding RNA, miR-122, was found to functon as development regulator and upregulate HCV RNA by interaction with the $5^{\prime} \mathrm{NCR}$. Then it turn out to be an attractive potantial new target for hepatitis $\mathrm{C}$ genetherapy.

The methods used as hepatitis C gene therapy include the use of ribozymes, antisense oligodeoxynucleotides, aptamers, decoys and RNA interference (RNAi). Several ribozymes targeting highly conserved HCV sequences were found to effectively degrade positive and negative strands of HCV RNA in vitro. Also, viral vectors to deliver and express ribozymes was evaluated for their substantial inhibition of $\mathrm{HCV}$ gene expression. Antisense 
oligonucleotides complementary to the HCV 5 'NCR has been evaluated for their effects on the expression and replication of HCV. In vitro study showed effective inhibition on HCV mRNA and unexpression HCV mRNA by antisense oligonucleotides at prety low concentrtation. The expression of $\mathrm{HCV}$ was also reduced specifically in the livers in a dose-dependent manner in BALB/c mice by specific antisense oligonucleotides. Using natural biological characteristic of RNAi targeting viral mRNA for degraation by cellular enzymes, RNAi might imply a potential therapitic strategy for hepatitis C genetherapy. Synthetic siRNA specific for HCV 5'NCR were disigned and observed for inhibition efficacy on HCV replication and protein synthesis. In the related research, effctive site in the $5^{\prime} \mathrm{NCR}$ was identified to achive more than $80 \%$ suppression of $\mathrm{HCV}$ replication at low concentration of specific siRNA. DNA-based vectors expressing siRNA against HCV were also investigated. By this methods, specific RNAi could be delivered into hepatocytes in vivo effectively.

\section{Impact of $\mathrm{HCV}$ genotype and treatment regime \\ Peter Ferenci \\ Medical University of Vienna, Austria}

Peginterferon alfa in combination with ribavirin is and will remain for the next years the current standard for treatment of chronic hepatitis $\mathrm{C}$. The new antivirals currently investigated in phase II of III trials may augment the overall response rates but require peginterferon/ribavirin as backbone.

The cure rate of peginterferon/ribavirin treatment depends on host and viral factors. Among the viral factors the genotype has the highest predictive value. Patients infected with genotype 2 have the highest cure rates $(>80 \%)$, followed by genotypes $3(70 \%)$ and $4(60 \%)$. The lowest cure rates are obtained in patients with genotype 1 (US: $45 \%$, Europe $>50 \%$ ). No large studies are available in patients with genotypes 5 and 6 . The genotype also determines treatment duration and the dose of ribavirin needed. Measuring the viral response at various time points during treatment allows individualization of treatment duration. Treatment duration may be shortened in patients with undetectable HCV-RNA after 4 weeks of therapy (rapid virologic response), on the other hand slow virologic responders may benefit from prolonged treatment. The dose of ribavirin can be reduced to $400-800 \mathrm{mg} /$ day in patients with genotypes 2 and 3 as long as they are treated for 2 weeks, whereas patients with genotypes 1 and 4 require full dose of ribavirin $(11.6 \mathrm{mg} / \mathrm{kg}$ bodyweight/day). Except for bodyweight and alcohol abuse, host factors cannot be modified. However response rates can be improved by better education of treating physicians to identify and treat conditions which negatively influence the final outcome of therapy. Specific focus is the prevention and/or early treatment of common side effects of therapy including anaemia, cytopenia and depression.

\section{Concurrent Session 2: Controversy in Guidelines of HBV \\ 14 Feb, 2009 (Saturday), 15:30 - 17:00 \\ Convention Hall B}

\begin{tabular}{l}
\hline Start Treatment for Low ALT - PRO \\
Nancy W.Y. Leung \\
Alice Ho Miu Ling Nethersole Hospital \& the Chinese University of Hong \\
Kong, Hong Kong SAR
\end{tabular}

Serum alanine aminotransferase (ALT) has been used as a surrogate marker for the degree of inflammation in liver diseases. It is a simple, readily available, and cheap blood test that can be repeated serially for monitoring disease progression. It has good correlation with the histological grading and staging of chronic hepatitis $\mathrm{B}(\mathrm{CHB})$. In the development of therapy for $\mathrm{CHB}$, the main endpoint was initially targeted at the normalization of abnormal ALT. In recent years, FDA added histological and viral response to ALT normalization as combined endpoints for assessing efficacy of treatment in clinical trials.

Almost all pivotal clinical trials on HBV therapy required raised ALT in the protocol inclusion criteria. Results from these trials formed the backbone for developing HBV consensus and guidelines such as AASLD, EASL, and APASL. This is evidence-based medicine. There is very little published controlled data on therapy for low or normal ALT among CHB patients. However, there are many good reasons to treat patients with low ALT.

1. ALT may fluctuate, some may have recurrent short ALT elevation or even flares. Higher levels are easily missed in usual clinical practice when ALT is monitored at 3 to 6 monthly interval.

2. The "normal" ALT used in different laboratories is above the new suggested normal of 30IU/L for males and 19IU/L for females.

3. Many recent research and studies indicate patients with "persistent" low or normal ALT have significant liver diseases that require therapy.

4. A percentage of CHB patients are in immune tolerant stage and have very high viral load but normal ALT. Antiviral therapy is not generally recommended for these patients, especially if they are young. However, many are in their fourth, fifth and even sixth decoade of life. Evidence suggests that this prolonged duration of viraemia exposes them to higher risk of fibrosis progression and hepatocelluar carcinoma development.

5. In $\mathrm{HBeAg}$ negative CHB, the ALT may be even more difficult to monitor. These patients are older and despite lower serum HBV DNA, have a higher probability of significant liver disease.

6. In patients with advance fibrosis or cirrhosis, even low rate of disease progression reflected by low ALT level may indicate insidious progression. Withholding therapy in these patients will hasten decompensation and death in short duration.

In summary, there is a strong case for treating CHB patients with low ALT, when there are options of effective and safe therapy. However, it must be emphasized that the recommendation to treat CHB patients with low ALT does not supersede a proper full assessment of the patient. Other factors that should prompt clinician to treat are the male gender, older age group, family history of serious liver disease or hepatocellular carcinoma, high serum HBV DNA levels, histological grading and staging or imaging suggesting coarse liver echo texture or cirrhosis.

\section{Start Treatment for Low ALT - Con}

Wan-Cheng Chow

Singapore General Hospital, Singapore

\author{
Nucleos(t)ide analogs can be stopped - PRO \\ Jidong Jia \\ Liver Research Center, Beijing Friendship Hospital, Capital Medical \\ University, Beijing, China
}

Chronic hepatitis B is a prevalent disease worldwide, especially in Asia. Long term follow-up studies show that the persistently exposure to high viral load is the major driving force for disease progression. Randomized clinical trials also demonstrate effective antiviral therapy can prevent the disease progression in chronic hepatitis B patients with advanced fibrosis or early cirrhosis. Therefore, all the major guidelines/consensus on the management of chronic hepatitis B emphasize the pivotal role of antiviral therapy.

Up to now, only interferons and nucleos(t)ide have been proved worldwide for the treatment of chronic hepatitis B and both of these 2 antiviral therapies can be used as first line therapy. The interferon-based therapy is advantageous in terms of finite duration, relatively higher $\mathrm{HBeAg}$ seroconversion rate and durable efficacy once the response has been achieved. On the other hand, the nucleos $(t)$ ide analogs possess potent antiviral activity, can be taken orally and less adverse effect. Not surprisingly, one argument against the use of nucleos(t)ide analogs is that long-term therapy is needed. Furthermore, this is sometimes misinterpreted as life-long therapy. This is not true in the following situations.

Firstly, chronic hepatitis B is an infectious disease, not a genetic disease. 
Therefore, life-long therapy is not required theoretically.

Secondly, clinical reports show that short duration of NAs is effective for those with refractory course or severe clinical manifestations. In this situation NAs can be stopped safely when clinical and viralogical response has been achieved. Another clinical scenario is the prophylactive use of NAs in patients who receive immune suppressive therapy. NAs can be stopped 6 weeks to 6 months after the completion of immune suppressive therapy.

Thirdly, even for chronic hepatitis B, clinical evidence shows that once the $\mathrm{HBV}$ DNA is suppressed for an extended period of time and $\mathrm{HBeAg}$ seroconversion has been achieved, NAs can be stopped after an 6 months of consolidation. In over $80 \%$ of these patients, the $\mathrm{HBeAg}$ conversion is stable for least one year. $\mathrm{HBeAg}$ seroconversion rate and their durability are determined by host factors such as age, gender, routine of HBV infection, immune status and liver disease activity, and virological profiles such as genotypes, $\mathrm{HBeAg}$ positivity and gene mutations. Therefore, clinicians should clearly understand all these baseline factors and carefully weigh these variables in deciding whether to treat a patient, what to use and for how long to treat. For patients of young age, $\mathrm{HBeAg}$ positive, HBV genotype A or B, high liver diseases activity (higher ALT and/or active necroinflammation in histology) and without cirrhosis, the $\mathrm{HBeAg}$ seroconversion rate is high and NAs can be stopped after 6 months of consolidation. For patients of older ages, $\mathrm{HBe} A g$ negative, genotype $\mathrm{C}$, low diseases activity and with cirrhosis, long-term nucleos(t)ide analog therapy would be preferred; however, even in this group of patients Nas can be stopped at least for some patients.

Nucleos(t)ide Analog Can Be Stopped - CON

Eugene R. Schiff

University of Miami School of Medicine, United States

Suppression of HBV DNA levels, ideally to undetectable, has been associated with marked histologic improvement of necroinflamation and regression of hepatic fibrosis. Long-term treatment is costly and fraught with compliance problems but the advantages of long term treatment outweigh the risk of discontinuing therapy. HBeAg disappearance followed by seroconversion requires consolidation therapy for at least a year to ensure "durability" of this response after discontinuation of the antiviral therapy. There is a lack of long term data supporting true durability and many of these patients continue to have detectable HBV DNA, albeit low viral levels. Chronic $\mathrm{HBeAg}$ negative hepatitis patients notoriously relapse when treatment is stopped even after years of HBV DNA negativity on therapy. In the minority of patients who lose HBsAg, durability of the HBV DNA negativity is undoubtedly more long lasting.

If the patient has underlying cirrhosis or is a high risk for hepatocellular carcinoma discontinuation of treatment cannot be justified. Fortunately more recently licensed drugs such as entecavir and tenofovir are very robust and the rate of evolving resistant mutations thus far is very low. Rapid attainment of HBV DNA negativity documented with sensitive assays is much less likely to result in clinically significant viral breakthrough among compliant patients. With periodic follow-up, which must be implemented in all HBV patients, compliance can be established and early recognition of those few with viral resistance accomplished.

There are many examples of diseases that require long-term indefinite therapy, e.g. hypercholesterolemia, hypertension, glucose intolerance. The primary limitation in long term antiviral therapy for HBV in 2009 is cost. Otherwise the beneficial effects of viral suppression are maximized with long term indefinite antiviral therapy.

\begin{tabular}{l} 
Concurrent Session 3: HCC Prevention and \\
Surveillance \\
14 Feb, 2009 (Saturday), 15:30 - 17:00 \\
Convention Hall C \\
\hline
\end{tabular}

Epidemiology of HCC in Asia Pacific

Man-Fung Yuen

The University of Hong Kong, Hong Kong SAR

Because of the high prevalence of chronic hepatitis B (CHB) in Asia Pacific region and chronic hepatitis $\mathrm{C}$ in the Western countries, primary liver cancer in particular hepatocellular carcinoma (HCC) remains a significant global disease. It is among the top 3 causes of cancer death in the Asia Pacific region. The incidence rate of $\mathrm{HCC}$ has been static for recent decades in Asia Pacific region. With older age being a major risk factor, the incidence increasing exponentially after age 40 years. Male has a higher incidence of HCC, with male to female ratio of 3:1, except in elderly Japanese with equal sex incidence or female predominance. CHB is the most common etiological agent accounting for $75-80 \%$ of cases in most Asia-Pacific countries. On the contrary, chronic hepatitis $\mathrm{C}$ infection accounts most of the cases in Japan, Singapore and Australia/New Zealand. The survival rate has improved slightly over years in spite of the major advances in surgery, liver transplantation and newer medical therapies. It is related to the fact that the majority of patients presents with advanced diseases, hence reducing the chance of curative treatment. With the expectation of declining CHB infection because of the implementation of universal hepatitis B virus vaccination in late 1980s in most of the Asia Pacific countries, the incidence of HCC may fall in the coming two to three decades. This may also be partly due to the decreased incidence of medical transmission of hepatitis $\mathrm{C}$ virus infection. However, some of the independent risk factors e.g. transmission of $\mathrm{HCV}$ by injection drug use, obesity and diabetes may partly offset this decline.

\section{Prevention of Hepatocellular Carcinoma}

\section{Seng-Gee Lim}

National University Health System, Singapore

Hepatocellular carcinoma $(\mathrm{HCC})$ is the $5^{\text {th }}$ common solid tumour cancer in the world and is ranked $3^{\text {rd }}$ in term of mortality, and is one of the most common cancers in Asia. HCC risk is related to the aetiology and liver disease status of patients. The most common cause of liver disease in Asia is chronic hepatitis B with the exception of Japan where chronic hepatitis C is the most common cause of liver disease. Other causes of liver disease include alcoholic liver disease, non-alcoholic steatohepatitis, autoimmune liver disease and metal deposition diseases. For chronic hepatitis B, the key initiative is prevention of chronic infection through a childhood vaccination program which has been implemented throughout Asia with variable levels of successful coverage. Evidence of successful prevention of $\mathrm{HCC}$ has been shown in Taiwan with the reduction in childhood HCC following a successful vaccination program. However, for those who already have chronic hepatitis B, the risk of HCC has been shown in the REVEAL study to be closely related to the level of HBV DNA. In addition, those with liver cirrhosis have shown to have a higher risk of HCC than non-cirrhotics. In the Asian lamivudine cirrhosis study, lamivudine was shown to successfully reduce the risk of progression of liver disease including HCC compared to placebo. However, it has not been demonstrated as yet that treatment of non-cirrhotics with high HBV DNA levels leads to a reduction in HCC. For those with chronic hepatitis $\mathrm{C}$, the risk of $\mathrm{HCC}$ is related to development of cirrhosis although non-cirrhotic $\mathrm{HCC}$ has been reported. Reduction of subsequent HCC has been shown. For other aetiologies of liver disease, reduction in HCC relies on treating and controlling the risk factors. Chemotherapeutic agents such as phytochemicals, anti-oxidants, herbal therapy and supplements have not been proven to prevent HCC. For patients at risk of HCC, regular screening strategies are important to detect early $\mathrm{HCC}$ but are not preventive measures.

In conclusion, $\mathrm{HCC}$ remains one of the most common Asian cancers and the main risk factor is chronic hepatitis B and C infection. Hepatitis B vaccination not only prevents chronic infection but also HCC development, and is highly cost effective. For those who already have chronic hepatitis B or $\mathrm{C}$ or other forms of liver disease, treatment and control will be needed to prevent HCC development. 
Surveillance Program For Hepatocellular Carcinoma

Deepak Amarapurkar

Bombay Hospital \& Medical Research Centro, Mumbai, India

Hepatocellular Carcinoma (HCC) is common cancer world wide and is a major public health problem in Asian Pacific region. HCC is a potentially viable target for surveillance as it occurs in well defined risk population (cirrhosis is a primary risk factor). Surveillance of HCC has been recommended by various organizations like AASLD, APASL. Surveillance has been practiced widely by gastroenterologists and hepatologists all over the world and it has become a standard practice eventhough evidence of its benefits has not yet been clearly established. Alfa feto protein (AFP) and/or ultrasound examination have been used every six monthly for surveillance programme. Usefulness of surveillance programme for early diagnosis of $\mathrm{HCC}$ in clinical practice has been shown in number of studies. In five retrospective studies comparing $\mathrm{HCC}$ detected on surveillance when compared with the HCC detected without surveillance, small and potentially curable HCCs were detected in surveillance group. But this surveillance programme did not improve prognosis in patients with advanced cirrhosis In surveillance groups HCCs detected are usually small relatively uniform. HCC in the no surveillance group are large and varied. HCC in the non surveillance group are detected in two different manners a) asymptomatic discovered outside the regular surveillance programme b) symptomatic. HCC detected without symptoms in non surveillance group are also smaller as compared to symptomatic group and the efficacy of surveillance programme will be decided on number of asymptomatic patients detected incidentally in non surveillance group. Cost effectiveness surveillance programme depends critically on the rate of small HCCs detected accidentally in non surveillance group, annual incidence of $\mathrm{HCC}$ in various etiologies of HCCs and adoption of liver transplantation as a treatment strategy. Surveillance programme detects small tumors and allows application of curative studies. The incremental cost effective ratio for six monthly AFP and ultrasound varies between USD 24,500 to 46,600 per QALY. Surveillance for HCC has been widely adopted but concrete evidence of cost effective benefit is not available. Though there have been more than 25 observational studies are available proving the superiority of surveillance programme over no surveillance. These studies have limitations of lead time bias, length bias for early diagnosis of small $\mathrm{HCCs}$, difference growth rate of $\mathrm{HCC}$ and poor compliance of the surveillance programme. Surveillance can be effective in reducing disease specific mortality with acceptable cost effectiveness among select group of patients. Cost effectiveness depends on rates of 1) rate of incidentally detected small HCC 2) annual incidence of HCC 3) adoption of transplant as a treatment strategy and 4) younger age of screen population. Conclusions from observational studies, computerized decision analytical models suggests that 1) ultrasound + AFP six monthly in a mixed etiology cohort is most effective surveillance strategy 2) cost effectiveness of surveillance strategy is dependent on etiology 3) much more cost effective in HBV related cirrhosis and least in the alcoholic cirrhosis.

\section{Recommendation on the Surveillance Program for HBV and HCV \\ Kwang-Hyub Han \\ Yonsei University College of Medicine, Seoul, Korea}

Chronic hepatitis B virus (HBV) or hepatitis C virus (HCV) infection is the most important risk factor in the development of hepatocellular carcinoma (HCC). Although persistent and progressive liver damage is a key mechanism of HCC development, there is some difference between HBV and $\mathrm{HCV}$ infection.

In hepatitis B carriers, the annual incidence of HCC is about $0.5 \%$. The incidence in cirrhotic patients is about $2.5 \%$ per year. In Asia, non-cirrhotic patients with $\mathrm{HBV}$ infection remain at risk for HCC. One factor that seems to predict the development of HCC is persistent viral replication. Several studies including REVEAL-HBV strongly suggested that serum HBV DNA concentration was the major risk factor. AASLD guideline recommends surveillance for HCC among not only HBV-related cirrhotic patients but also those at an older age, with family history of HCC, or with high serum HBV DNA level.

A model to predict the risk of $\mathrm{HCC}$ in HBV-positive population will help follow and counsel individual patient. In our study of $688 \mathrm{HBsAg-positive}$ patients who had underwent surveillance with ultrasonography (US) and serum alpha-fetoprotein (AFP), 46 developed HCC during mean of 50 months. Multivariate analysis showed that age over 40 years, male gender, baseline serum $\mathrm{AFP} \geq 20 \mathrm{IU} / \mathrm{ml}$, echo pattern of severe coarseness, habitual alcohol ingestion were independently associated with HCC development. Increasing evidences suggest that persistent $\mathrm{HBV}$ replication indicated as serum HBV DNA concentration is a predictor of HCC. In addition, suppression of HBV replication by oral nucleotide analogue resulted in lower incidence of HCC compared to no treatment.

The HCC related to chronic hepatitis $\mathrm{C}(\mathrm{CHC})$ is a major concern in USA, Europe, and Japan. In a Japanese study, serum AFP $(\geq 20 \mathrm{ng} / \mathrm{ml})$, male gender, age ( $\geq 55$ years), and platelet count $\left(<100,000 / \mathrm{mm}^{3}\right)$ were found to be independently associated with $\mathrm{HCC}$ development. However, HCC rarely occurs in anti-HCV positive patients without cirrhosis. In a prospective population-based study, anti-HCV-positive subjects had a 20 -fold increased risk of HCC compared with anti-HCV-negative subjects. The incidence of $\mathrm{HCC}$ in cirrhotic patients with $\mathrm{HCV}$ infection is between $2 \%$ and $8 \%$ per year. Non-cirrhotic patients with HCV infection have a lower risk of HCC development. However, the onset of cirrhosis cannot be accurately determined.

Since advanced hepatic fibrosis is more correlated with HCC development, simple and non-invasive assessment of liver fibrosis is more implicated in chronic viral infection. Thus, a large scale study is needed to validate these non-invasive methods such as liver stiffness measurement (Fibroscan ${ }^{\circledR}$ ) or fibrotest in the prediction of HCC development.

The surveillance of HCC in high-risk patients proved to be cost-effective, especially in high endemic area. Most experts agree that US and serum AFP measurement every 6 months is the most appropriate method in the surveillance of HCC. However, this method is not satisfactory yet because of the low sensitivity. To enhance the effectiveness of surveillance program in Asia, alternative method or identification of a novel biomarker may be required.

\section{Concurrent Session 4: HBV Treatment 15 Feb, 2009 (Sunday), 15:30 - 17:00 Convention Hall A}

How to Choose between Peg-interferon and Nucleoside Analogues

Harry L.A. Janssen

University Medical Center, Rotterdam, The Netherlands

When deciding on the antiviral drug to be given, several factors have to be taken into account. The major advantage of PEG-IFN is the higher chance of achieving sustained response compared to nucleos(t)ide analogues with a finite duration of therapy. Disadvantages are the subcutaneous administration and the frequent occurrence of side effects. The major advantage of nucleos $(\mathrm{t})$ ide analogues are the favourable tolerability and the oral administration. Disadvantages are the long duration of therapy and the subsequent risk of antiviral resistance. The costs of a one-year course of nucleos(t)ide analogue therapy are lower than of PEG-IFN, but will easily be higher when long-term therapy is needed.

PEG-IFN can be considered as therapy in eligible patients because of the higher chance of achieving sustained off-treatment response compared to nucleos(t)ide analogues. Sustained transition to the immune-control phase (inactive HBsAg carrier state) can be achieved in $30 \%$ of $\mathrm{HBeAg}$ positive patients and $25 \%$ of $\mathrm{HBeAg}$ negative patients treated with PEG-IFN, implicating that treatment induced response is sustained in about $85 \%$ and $50 \%$ of $\mathrm{HBeAg}$ positive and $\mathrm{HBeAg}$ negative patients, respectively. Relapse occurs in at least $40 \%$ and $90 \%$ of $\mathrm{HBeAg}$ positive and $\mathrm{HBeAg}$ negative patients after discontinuation of nucleos $(t)$ ide analogue therapy, respectively. HBeAg-positive patients with a high chance of response to PEG-IFN therapy are those with genotype $\mathrm{A}$, and those with genotype $\mathrm{B}$ or $\mathrm{C}$ having serum HBV DNA below $1.0 \times 10^{9}$ copies $/ \mathrm{ml}\left(2.0 \times 10^{8} \mathrm{IU} / \mathrm{ml}\right)$ and serum ALT above twice the upper limit of normal. For HBeAg-negative disease the role of genotype is less well defined. However, it has been suggested that in 
HBeAg-negative patients genotypes B and C were more likely to achieve a sustained virologic response than genotype D. Furthermore, low serum HBV-DNA and high ALT levels are positive predictors of response in these patients. The licensed duration of peginterferon therapy is one year for both $\mathrm{HBeAg}$ positive and $\mathrm{HBeAg}$ negative chronic hepatitis B. However, the optimal duration of PEG-IFN therapy has not been established. In HBeAg positive patients, response rates after 24-32 weeks of treatment course seem comparable to those observed after one year, but head-to-head comparison is not available. Since early prediction of response to PEG-IFN is difficult in chronic HBV infected patients, the recommended the duration of therapy is still one year for all patients.

Nucleos(t)ide analogue therapy should be considered in all patients not responding to or not eligible for PEG-IFN therapy. This includes also patients with autoimmune disease, pre-existent psyciatric disorders or advanced cirrhosis (signs of diminished liver function or portal hypertension). When choosing a nucleos(t)ide analogue, potency and risk of resistance play an important role. It is recommended to choose those antivirals with most potency, highest genetic barrier, least toxicity.

\section{Is Combination Treatment Superior to Monotherapy?}

Anna S. F. Lok

University of Michigan, Ann Arbor, MI, USA

Substantial advances have been made in the treatment of chronic hepatitis B in the past decade. Currently, there are 7 approved therapies: 2 formulations of interferon (standard and pegylated) and 5 nucleos/tide analogues: lamivudine, adefovir, entecavir, telbivudine and tenofovir. In the vast majority of patients, these treatments are administered as monotherapies and a second drug is added when there is evidence of treatment failure. Many experts have advocated the use of de novo combination therapy citing the improvement in sustained virologic response rate when ribavirin is added to interferon for chronic hepatitis $\mathrm{C}$ and reduction in antiviral resistance rate when combination therapy is used for human immunodeficiency virus infection.

Several clinical trials have compared combination of pegylated interferon and lamivudine with pegylated interferon or lamivudine monotherapy. In each of these trials, combination therapy resulted in more marked on-treatment virus suppression compared to monotherapies but sustained virus suppression (and $\mathrm{HBeAg}$ seroconversion in the case of $\mathrm{HBeAg}$-positive patients) after discontinuation of treatment was similar to pegylated interferon monotherapy. Combination therapy was associated with a lower rate of antiviral resistance compared to lamivudine monotherapy but this benefit should be interpreted in the context of zero resistance in patients who received pegylated interferon monotherapy. These trials demonstrated that de novo combination therapy of pegylated interferon and lamivudine does not confer an advantage over pegylated interferon monotherapy. One pilot trial reported very promising results with de novo combination therapy of pegylated interferon and adefovir but the number of patients enrolled was small and the study did not include control groups who received monotherapies.

There have been very few clinical trials comparing de novo combination of nucleos/tide analogues. The largest trial compared combination of lamivudine and adefovir vs. lamivudine alone. Combination therapy did not result in more rapid virus suppression. Although combination therapy was associated with a lower rate of resistance to lamivudine, a resistance rate of $15 \%$ after 2 years treatment was unacceptably high in light of resistance rates of $0-1 \%$ with entecavir or tenofovir monotherapies. Another small trial reported that combination of emtricitabine and adefovir resulted in more marked virus suppression than adefovir alone, but this trial did not include an emtricitabine alone group and the "additive" antiviral activity was likely an artifact related to the weak antiviral activity of adefovir monotherapy. In summary, there is sound basis for de novo combination therapy of chronic hepatitis B but data to date do not support its use clinically, particularly in counties where antiviral drugs with high genetic barrier to resistance are readily available.
How will Tenofovir Change the Choice of Anti-viral Agents

Patrick Marcellin

Hopital Beaujon, Clichy, France

Background: Tenofovir disoproxil fumarate (TDF) has activity against hepatitis $\mathrm{B}$ virus and was recently approved for the treatment of CHB. Methods: Patients with HBeAg-positive (+) or -negative (-) CHB were randomized 2:1 to double-blind, once daily TDF $300 \mathrm{mg}$ or adefovir dipivoxil $10 \mathrm{mg}(\mathrm{ADV})$. After 48 weeks, patients rolled to open-label (OL) TDF for an additional 7 years with the option to initiate combination emtricitabine (FTC) + TDF treatment at or after W72 for confirmed HBV DNA $\geq 400$ copies (c) $/ \mathrm{mL}$ (69 IU/mL). Results: HBeAg+: At W96, $86 \%$ $(228 / 266)$ remained on study. Viral suppression on ADV was maintained/enhanced after rolling to TDF (ADV-TDF): $100 \%$ of stable and $82 \%$ viremic patients on $\mathrm{ADV}$ had HBV DNA $<400$ copies (c) $/ \mathrm{mL}$ at $\mathrm{W} 96$. In an intent-to-treat (ITT) analysis, $78 \%$ of ADV-TDF patients had HBV DNA $<400 \mathrm{c} / \mathrm{mL}$ at W96. Patients treated with TDF for 96 weeks (TDF-TDF), 78\% (ITT) had HBV DNA $<400 \mathrm{c} / \mathrm{mL}$ at W96 (89\% observed). Twenty-eight patients initiated OL FTC+TDF before W96 for HBV DNA $\geq 400 \mathrm{c} / \mathrm{mL}$. Overall 27 patients (15 TDF-TDF; 12 ADV-TDF) had HBV DNA $\geq 400 \mathrm{c} / \mathrm{mL}$ at W96. Twenty-seven percent (27\%) (TDF-TDF) and $22 \%$ (ADV-TDF) HBeAg seroconverted $(\mathrm{p}=0.31)$. Six percent $(6 \%)$ in both groups experienced HBsAg loss (11/15 had anti-HBs). Mean ALT was similar at W96 between groups ( $36 \mathrm{U} / \mathrm{L})$. One patient discontinued due to an adverse event $(\mathrm{AE})$ : mild, transient increase in serum creatinine (peak 1.3 $\mathrm{mg} / \mathrm{dL}$ ). HBeAg-: At W96, 89\% (335/375) remained on study. Viral suppression on ADV was maintained/enhanced after rolling to TDF (ADV-TDF): $100 \%$ of stable and $100 \%$ viremic patients on ADV were $<400$ $\mathrm{c} / \mathrm{mL}$ at W96. $89 \%$ of ADV-TDF patients had HBV DNA $<400 \mathrm{c} / \mathrm{mL}$ at W96 (ITT). Patients treated with TDF for 96 weeks (TDF-TDF), 91\% (ITT) had HBV DNA $<400 \mathrm{c} / \mathrm{mL}$ at $\mathrm{W} 96$ ( $99 \%$ observed). Two patients had HBV DNA $\geq 400 \mathrm{c} / \mathrm{mL}$ at W96. Two patients initiated OL FTC+TDF for HBV DNA $\geq 400 \mathrm{c} / \mathrm{mL}$. Mean ALT was similar at $\mathrm{W} 96$ between groups (ALT=35 U/L). Two patients discontinued due to AEs: hepatocellular carcinoma in one and fatigue/lack of concentration/dizziness in the other patient. One patient died of cholangiocellular carcinoma during year 2. Conclusion: TDF produced potent, continuous viral suppression and was well tolerated through week 96 . Patients rolling to TDF after 48 weeks of ADV treatment benefited with significant additional viral suppression and had a similar response to patients treated with TDF for 96 weeks.

\section{Is There any Loophole in the Roadmap Model?}

Edward J. Gane

New Zealand Liver Transplant Unit, New Zealand

In large observational, longitudinal studies of the natural history of chronic hepatitis B (CHB), high levels of HBV replication are associated with increased risk of cirrhosis, decompensation, hepatocellular carcinoma and liver related mortality. In addition, long-term studies of antiviral therapy for CHB have demonstrated that sustained suppression will prevent disease progression and complications. Profound and sustained inhibition of viral replication is therefore the most important goal of antiviral therapy in chronic hepatitis B. Multiple analyses of baseline factors and on-treatment responses have identified that the absolute HBV DNA level after 24 weeks of therapy as the best predictor of long-term efficacy (normal ALT, HBV DNA nondetectability, lack of resistance). Patients with undetectable serum HBV DNA levels after 24 weeks have the best long-term outcomes whilst those with levels remaining above 10,000 copies per $\mathrm{ml}$ are unlikely to benefit from long-term therapy with that particular agent and either the addition or switch to another antiviral agent with increased potency but without cross resistance could be considered at this time point.

This on-treatment management algorithm where virologic responses at 24 weeks are used to identify those patients with suboptimal responses so that an appropriate change in therapy can be initiated in order to enhance long-term patient outcomes is called the "Roadmap Concept".

There are several potential loopholes in the practical application of this approach: 
(i) The Roadmap Concept is perhaps best suited to agents with medium or low genetic barrier to resistance such as Lamivudine and Telbivudine, where continued therapy in patients with suboptimal early viral suppression is associated with very high rate of resistance with subsequent virologic breakthrough and disease progression. In contrast, the 24 week serum HBV DNA level is a poor predictor for long-term efficacy in antiviral agents with high genetic barrier to resistance such as Entecavir or Tenofovir, which are both associated with extremely low rates of virologic breakthrough $(0.8 \%$ after 5 years Entecavir; $0 \%$ after 2 years Tenofovir). For example, $79 \%$ of patients who remain PCR detectable after 24 weeks Tenofovir will achieve PCR nondetectability by 76 weeks (Heathcote et al, EASL 2008) compared to This is not however virologic breakthrough. In contrast,

(ii) There is a lack of evidence to support which is the best strategy for partial and inadequate responders to lamivudine or Telbivudine. If the treatment is to be changed, which new agent should be used and should this be switched or added on to the original agent? Although the addition of a second agent should prevent emergence of resistance to either agent alone, there is no data to suggest that this will improve viral suppression overthat achieved by switching to the second agent.

(iii) This approach is unlikely to be suitable for previously treated patients because of the risk of accelerated emergence of resistance to subsequent agents.

(iv) This approach is unlikely to be applicable with immunomodulatory therapies including standard and pegylated interferons.

(v) Finally, there has been no pharmaco-economic modelling performed to determine whether the Road Map approach (initial therapy with an agent with low genetic barrier to resistance and intensifying therapy after 24 weeks if serum HBV DNA remains detectable) is more cost-effectiveness than starting with an agent with high genetic barrier to resistance and ignoring the 24 week serum HBV DNA level.

The most attractive application of the Road Map approach would be initial therapy in HbeAg positive CHB with an antiviral agent, which despite a low genetic barrier, achieves superior HbeAg seroconversion rate compared to other agents. Further studies are needed to determine whether Telbivudine will be such an agent.

\section{Concurrent Session 5: Liver Fibrosis 15 Feb, 2009 (Sunday), 15:30 - 17:00 Convention Hall B}

\author{
Non-invasive Assessment of Liver Fibrosis \\ Gui-Qiang Wang \\ Peking University First Hospital, Beijing, China
}

Hepatic fibrosis is an important feature of progressive chronic liver diseases, and the prognosis and management of chronic liver diseases depend on the degree of liver fibrosis. Therefore, the assessment of liver fibrosis is crucial for predicting disease progression and for treatment planning, especially for the antiviral treatment in chronic hepatitis B. Liver biopsy is the current gold standard for the diagnosis of liver fibrosis, but is an invasive and cannot be repeated often and difficult to be accepted by both physicians and patients. Some alternative non-invasive methods have been developed, including serum markers with different models such as Fibrotest and Fibrometer, the transient elastography (Fibroscan), Magnetic resonance (MR) elastography, and ultrasound elastography. Fibrotest and Fibrometer and other direct and indirect serum markers can predict the presence of significant fibrosis or cirrhosis in patients with chronic liver disease with considerable accuracy. Transient elastography is a new method for the evaluation of liver stiffness based on changes in tissue elasticity induced by hepatic fibrosis and is a noninvasive, reproducible and reliable method to assess hepatic fibrosis as well as to diagnose liver cirrhosis. Magnetic resonance (MR) elastography, a technique for quantitatively assessing the mechanical properties of soft tissues, has shown a safe, noninvasive technique with excellent diagnostic accuracy for assessing hepatic fibrosis. It seems that all non-invasive assessment of liver fibrosis have a good diagnostic accuracy to discriminate patients with mild fibrosis to those with severe fibrosis, but for intermediate stages of liver fibrosis show discordance. A combination of different measurement may improve the diagnostic accuracy and the new non-invasive markers need to be developed.

\section{Reversibility of Liver Fibrosis \\ Teerha Piratvisuth \\ Prince of Songkla University, Thailand}

Hepatic fibrosis is the common consequence of a reaction occurring in response to liver injury and inflammation. Chronic liver injury results in fibrosis progression leading to liver cirrhosis. With the current available therapy for chronic liver disease one of the important question is whether liver fibrosis can be reversible. Regression or reversion of fibrosis after discontinuation of the cause of liver injury or following treatment with an antifibrotic agent has been demonstrated in animal studies. Activated hepatic satellate cells in chronic liver injury are the major source of fibrillar extracellular matrix as well as of the tissue inhibitors of metalloproteinases, which inhibit collagen degradation. Apoptosis of activated hepatic satellate cells occurs after recovery from acute and chronic liver injury. Apoptosis of hepatic satellate cells and presence of macrophage cells could be responsible factors for degradation of collagen resulting in fibrosis regression. Evidence of fibrotic or cirrhotic regression in human has been reported in chronic liver diseases of different etiologies including viral hepatitis. Improvement in liver fibrosis after longtern anti HBV treatment has been shown in many studies which have paired liver biopsy. $63 \%$ of lamivudine treated patients had improvement of bridging fibrosis after 2 years of lamivudine therapy. Fibrosis improvement was also seen in $60 \%$ of patients after 5 years of adefovir dipivoxil therapy. Longterm entecavir therapy for 6 years shew at least 2-point decrease in Ishak fibrosis score in $58 \%$ of the patients. Serpaggi etal reported disappearance of cirrhosis, defined as a decrease of 2 or greater in METAVIR fibrosis score in 14 $(12.4 \%)$ of the 113 cirrhotic patients who received specific therapy. Poynard also confirmed the reversal of cirrhosis in 75 (49\%) of 153 chronic hepatitis C patients with base-line cirrhosis who were treated with Peginterferon with or without ribavirin. Liver biopsy-proven cirrhosis reversion has been shown in patients with chronic hepatitis B after longterm lamivudine therapy. Non-invasive methods for evaluation of liver fibrosis, such as fibro-test, fibroscan etc, may be useful for monitoring fibrosis regression in patients with chronic liver disease who undergoing specific therapy. However, study to validate the use of these non-invasive methods in monitoring fibrosis regression is essential before applying into the clinical practice.

\section{Reactive Oxygen Species and Liver Fibrosis \\ Ramón Bataller \\ Hospital Clinic de Barcelona, Spain}

Reactive oxygen species (ROS) play an important role in hepatic fibrosis. Increased ROS are commonly detected in livers from patients with alcohol abuse, hepatitis $\mathrm{C}$ virus infection, iron overload or chronic cholestasis, as well as in most types of experimental liver fibrogenesis. Moreover, antioxidant agents attenuate hepatic fibrosis in rodents and in patients with chronic liver diseases. The cellular origin and molecular mechanisms underlying the fibrogenic effect of ROS are under investigation. CYP2E1-derived ROS from damaged hepatocytes can induce phenotypic activation, proliferation and increased collagen synthesis in cultured Hepatic stellate cells (HSC). An alternative source of ROS in the injured liver is NADPH oxidase. This enzyme is present in Kupffer cells and generates large amounts of superoxide anions in alcohol-induced liver disease. Moreover, fibrogenic mediators such as angiotensin II stimulates a non-phagocytic form of NADPH oxidase in HSC, activating redox-sensitive intracellular pathways. Besides increased ROS production, reduced removal of ROS by antioxidant enzymes is observed in advanced fibrosis. 


\section{Intestinal Permeability, Innate Immunity and Liver Fibrosis} David A Brenner

University of California, San Diego, School of Medicine, United States Background: The pattern recognition receptor Toll-like receptor (TLR) 4 plays a central role in innate immune responses. In hepatic fibrosis, portal level of the TLR4 ligand lipopolysaccharide (LPS) is elevated increased intestinal permeability. Both Kupffer cells, the resident macrophages in the liver, and hepatic stellate cells (HSCs), which play a crucial role in hepatic fibrosis, express TLR4. However, the precise contribution of TLR4 in hepatic fibrosis is unknown.

Aim: (1) To define the role of TLR4 in hepatic fibrosis and hepatocellular cancer (2) To identify the source of TLR4 ligand during hepatic fibrosis (3) To determine the cellular target of TLR4 ligand in the liver (4) To characterize molecular mechanisms by which TLR4 promotes HSC activation and fibrogenesis.

Methods: Hepatic fibrosis was induced by bile duct ligation (BDL) of TLR4-intact $(\mathrm{C} 3 \mathrm{H} / \mathrm{HeOuJ})$, TLR4-mutant $(\mathrm{C} 3 \mathrm{H} / \mathrm{HeJ}), \mathrm{MyD}^{-/-}$and $\mathrm{TRIF}^{-/-}$ mice. To deplete intestinal micro flora, we treated the mice with an antibiotics cocktail (Ampicillin, Metronidazole, Neomycin and Vancomycin) for 4 weeks prior to BDL. TLR4-chimeric mice were generated by $\mathrm{Cl}_{2} \mathrm{MDP}$ injection, irradiation and bone marrow (BM) transplantation of TLR4-mutant BM into TLR4-intact mice and vice versa. Hepatocellular cancer was induced in TLR4-intact and TLR4 mutant mice by a single injection of DEN. We evaluated hepatic fibrosis by sirius red staining, measurement of hydroxyproline content and profibrogenic gene expression (collagen 1(I),

SMA, TGF 1, TIMP1) by quantitative real time PCR (qPCR). Cellular targets of LPS in the liver were analyzed by nuclear translocation of NF- $\kappa$ Bp65 by immunofluorescence. To evaluate HSC activation, we measured collagen promoter activity in HSCs isolated from collagen-promoter driven GFP transgenic mice and TGF $\beta$ transcriptional activity after stimulation with TGF $\beta$ or co-culturing with Kupffer cells (KCs) LPS targets in HSCs were analyzed by microarray and qPCR. Results: Upon BDL, hepatic collagen and hydroxyproline content were less increased in TLR4-mutant mice and antibiotics-treated mice compared with TLR4-intact mice. Combination of $\mathrm{Cl}_{2} \mathrm{MDP}$, irradiation and BMT completely reconstituted $\mathrm{KCs}$, but not $\mathrm{HSCs}$, with $\mathrm{BM}$-derived cells, generating TLR4-chimeric mice. Chimeric mice containing TLR4-mutant BM/TLR4-intact HSC had normal fibrosis after BDL, whereas chimeric mice with TLR4-intact BM/TLR4-mutant HSC had reduced fibrosis, indicating HSCs are the main target of TLR4 ligand in hepatic fibrosis. After LPS injection into KC-depleted mice, NF- $\kappa \mathrm{B}$ was strongly activated in HSCs. LPS treatment enhanced HSC activity by TGF $\beta$ - and KC-induced TGF transcriptional and collagen promoter activity. Microarray analysis revealed 154 LPS-regulated genes in quiescent HSCs including downregulation of the TGF pseudoreceptor BAMBI. BAMBI was downregulated by LPS stimulation in WT-HSCs, but not in MyD88 $8^{-/}$or I B super-repressor-treated cells. TGF $\beta$ transcriptional and collagen promoter activities were upregulated and downregulated in the HSCs overexpressing of dominant negative BAMBI and full length BAMBI, respectively. Upon BDL, profibrogenic gene expression was suppressed in $\mathrm{MyD} 88^{-/-}$mice, but not in $\mathrm{TRIF}^{-/}$mice. TLR4 wild-type mice had more HCCs than TLR4 mutant mice. Conclusion: Hepatic fibrosis and HCC is induced by TLR4-dependent manner. HSCs, but not KCs are the main targets of TLR4 ligands in hepatic fibrosis. TLR4 enhances hepatic fibrosis by downregulating TGF pseudoreceptor BAMBI and enhancing TGF $\beta$ signaling in HSCs. In the fibrotic liver, myofibroblasts and fibrocytes are recruited from the bone marrow, but only constitute a small component of the Type I collagen expressing cells

\section{Concurrent Session 6: Virology \\ $15 \mathrm{Feb}, 2009$ (Sunday), 15:30 - 17:00 \\ Convention Hall C}

\author{
The Role of HCV RNA Monitoring \\ Ming-Lung Yu \\ Kaohsiung Medical University Hospital, Kaohsiung
}

Pegylated interferon-alpha (Peg-IFN) plus ribavirin is the current standard of care of chronic hepatitis $\mathrm{C}(\mathrm{CHC})$. For patients infected with $\mathrm{HCV}$ genotype 1 (HCV-1), the recommended treatment duration is 48 weeks, whereas for patients infected with $\mathrm{HCV}-2 / 3$, the recommended treatment duration is 24 weeks.

Recent studies on viral kinetics showed that on-treatment virological responses assessed by HCV RNA monitoring during therapy have been the most important predictors associated with the response to Peg-IFN/ribavirin. A rapid virological response at week 4 (RVR), defined as PCR-negative of serum HCV RNA at 4 weeks of treatment, is the single best positive predictor of a sustained virological response (SVR) to Peg-IFN-ribavirin for both HCV-1 and HCV-2/3 patients with a high degree of accuracy of prediction. In contrast, early virological response (EVR), defined as PCR-seronegative of HCV RNA or decrease of HCV RNA by 2 logs from baseline values after 12 weeks of therapy, is an even more robust negative predictor. The likelihood of an SVR is approximately $0 \%$ to $2 \%$ in cases without an EVR for HCV-1 patients. Thus it is recommended that HCV-1 patients who do not achieve an EVR should stop the treatment. More recently, EVR has been suggested to be subdivided into RVR, complete EVR (no RVR, but HCV RNA $<50 \mathrm{IU} / \mathrm{ml}$ at week 12) and partial EVR (HCV RNA $>2$ log drop in HCV RNA but still detectable [ $>50 \mathrm{IU} / \mathrm{ml}]$ at week 12) to further improve the prediction of patients who are likely to achieve an SVR and may allow for tailoring of treatment duration.

Individualized therapy has become a major consideration for clinicians. It is desirable to expose $\mathrm{CHC}$ patients to the lowest doses and shortest durations of treatment possible to reduce the likelihood of adverse events and to minimize costs, without compromising treatment efficacy for the rapid responders. On the other hand, some difficult-to-treat patients have to receive longer and/or higher dose therapy to achieve responses. Recently, several studies have demonstrated that shorter a 24-week regimen for $\mathrm{HCV}-1$ patients with lower viral loads and a RVR at week 4 were as effective as the current recommendations 48 -week treatment. Studies investigating shorter treatment durations for $\mathrm{HCV}-2 / 3$ patients with a RVR, however, did not observe consistent results because the shorter therapy arm and the dose of ribavirin varied across different studies. Nevertheless, a recent meta-analysis demonstrated only optimal shorter therapy (16 weeks with weight-based, standard dose of ribavirin), but not suboptimal shorter therapy (12-14 weeks or low dose ribavirin, $800 \mathrm{mg} / \mathrm{d}$ ) could provide efficacy equal to a standard 24-week treatment in HCV-2/3 patients with a RVR. Meanwhile, an extended 72-week regimen with weight-based, standard dose of ribavirin could improve the treatment efficacy for slow responders (pEVR) of HCV-1 patients, but not in cEVR patients. HCV genotype and HCV RNA monitoring during therapy could provide information for individualized therapy decision making for $\mathrm{CHC}$ patients to maximize the efficacy with optimal regimen.

\section{The Role of HBV DNA Monitoring}

Jean-Michel Pawlotsky

Hôpital Henri Mondor, Université Paris, Créteil, France

In a chronic $\mathrm{HBV}$ carrier, chronic hepatitis B is defined by an elevated serum HBV DNA. The goal of chronic hepatitis B therapy is to prevent progression of liver disease to its complications. This can be achieved if HBV replication is durably abolished or significantly reduced. Antiviral treatment is currently recommended in patients with an HBV DNA titer above 2,000 IU/mL. HBV treatment monitoring is based on HBV DNA quantifications and ALT determinations every 3 to 6 months, whatever the HBe serostatus and antiviral treatment. In $\mathrm{HBeAg-positive} \mathrm{patients,} \mathrm{treatment} \mathrm{efficacy} \mathrm{is}$ witnessed by the loss of $\mathrm{HBeAg}$ with may be followed by a seroconversion to anti-HBe antibodies. It is generally associated with a profound reduction of serum HBV DNA levels and normalization of aminotransferase activity. Ideally in HBe seroconverters, HBV DNA should be undetectable with a sensitive real-time PCR assay (lower limit of detection of the order 10 to 15 $\mathrm{IU} / \mathrm{mL}$ ) and aminotransferase activity should be normal. In HBeAg-positive patients with no $\mathrm{HBe}$ seroconversion and in $\mathrm{HBeAg}$-negative patients, the goal of antiviral treatment is to achieve a profound and durable HBV DNA suppression. The HBV DNA level should be undetectable on treatment with a sensitive real-time PCR assay (lower limit of detection: 10 to $15 \mathrm{IU} / \mathrm{mL}$ ). If 
the HBV DNA level remains detectable after 48 weeks, a second antiviral compound with no cross-resistance with the first one must be added in order to prevent subsequent resistance. In the patients who have responded and have been compliant, resistance should be suspected if the HBV DNA level rises by more than $1 \log _{10} \mathrm{IU} / \mathrm{mL}$ above nadir in two consecutive samples taken one month part. The identification of selected amino acid substitutions known to be associated with resistance to the administered drug(s) by means of molecular testing can help guide treatment adaptation. Consensus decisional algorithms will need to be established before systematic genotypic resistance testing can be recommended to adapt the treatment strategy to the resistance profile of the infecting viral strain.

\section{The Role of HBV Resistance Monitoring}

Scott Bowden

Victorian Infectious Diseases Reference Laboratory, North Melbourne, Victoria, Australia

The introduction of orally available nucleoside and nucleotide analogue inhibitors of the hepatitis B virus (HBV) polymerase/reverse transcriptase (NRTI) has resulted in spectacular improvements in the treatment of chronic hepatitis $\mathrm{B}(\mathrm{CHB})$ infection during the past decade. In developed countries, NRTI are now widely used to treat CHB due to their convenience, safety and efficacy. Unfortunately, because of the unusual replication strategy used by HBV, NRTI are virustatic rather than virucidal. Consequently, in the long-term, development of viral resistance is common and is becoming a major cause of treatment failure. Viral breakthrough associated with selection of antiviral-resistant HBV usually precedes biochemical breakthrough, clinical deterioration and progressive liver failure. Professional bodies, including AASLD, APASL and EASL, have published guidelines and recommendations for the management of NRTI-resistant CHB NRTI can be classified into subgroups based on structure and because resistance to different classes of NRTI does not usually confer significant cross resistance to other classes, resistance can be managed by switching therapy or continuing therapy by adding additional NRTI.

Measurement of viral load is indispensable for monitoring and confirming the presence of NRTI resistant virus, since nearly all instances of resistance to NRTI are initially identified by a sustained rise in viral load that occurs despite continuing antiviral therapy. The sensitive HBV DNA assays that are currently in use will eventually detect rising viral loads due to drug resistant virus even when the emergence of the drug resistant $\mathrm{HBV}$ population is slow. Since factors other than drug resistance (for example, poor patient compliance) can affect viral load, it cannot be automatically assumed that rising loads are indicative of drug resistance, which requires confirmation by other methods. Drug resistant HBV can be detected by genotyping and/or phenotyping, but only the latter provides direct measurements of in vitro resistance to specific drugs. Each of the currently available methods has inherent advantages and disadvantages.

Many obstacles need to be overcome for the therapeutic control of hepatitis $B$ in developing countries. NRTI use may be unfunded or sub-optimal NRTI therapy may be used because of lack of resources. This may lead to more rapid development of resistant virus which may preclude rescue therapy by more active agents. Economic factors may also delay detection of early resistance because of the inability to monitor HBV DNA levels. Finally, the identification of the pattern of the resistance changes in the HBV polymerase/reverse transcriptase is also important because it indicates the other classes of drugs which may be effective. However, this too requires funding. These problems need to be addressed if we are to reduce the development of resistant virus and to limit its potential transmission.

\section{The Role of Quantitative HBsAg Measurement Jinlin Hou \\ Nanfang Hospital, Nanfang Medical University, Guangzhou, China}

Hepatitis B surface antigen (HBsAg) loss under antiviral therapy is rare in chronic hepatitis B patients and the dynamics of serum HBsAg in these patients are not available. The changes in serum HBsAg following treatment with adefovir or peg-interferon- $\alpha-2 \mathrm{a}$ were studied in hepatitis B e-antigen (HBeAg) positive chronic hepatitis B patients. Abbott Architect HBsAg assay was used to quantify serum HBsAg. HBsAg levels were significantly decreased during the first 12 weeks of treatment with median change of $-397.0 \mathrm{IU} / \mathrm{ml}$ and $-555.4 \mathrm{IU} / \mathrm{ml}$ respectively for adefovir and peg-interferon- $\alpha-2 \mathrm{a}$ ( $\mathrm{p}=0.005$ and 0.001 respectively). Beyond 12 weeks, no further significant HBsAg reductions were found even in patients with sustained viral replication inhibition in either group. Three distinct patterns of HBsAg changes were observed in most patients in both treatment groups: biphasic pattern (rapid HBsAg reduction from baseline to week 12); assurgent pattern (higher HBsAg level at week 12 than at baseline); and wavy pattern (HBsAg reduction from baseline to week 12, followed by relapse at week 24 or week 28). These results might offer insights into the possible mechanism(s) underlying the unusual occurrences of HBsAg loss under antiviral therapy.

The influences of HBV genotype $(\mathrm{Ba} / \mathrm{C})$ on serum and intrahepatic $\mathrm{HBsAg}$ levels were further studied. Serum HBsAg was measured with Abbott Architect i2000 and HBV genotype was determined by restriction fragment length polymorphism combined direct sequencing in 469 chronic hepatitis B patients. Immunostain for HBsAg was carried out in 118 patients. Percentages of synthesized HBsAg detained in hepatocytes were compared among four common HBV genotypes in transfection experiments. Sequencing for large surface gene was performed in 47 patients with distinct intrahepatic $\mathrm{HBsAg}$ expressions. Serum $\mathrm{HBsAg}$ levels were higher in $\mathrm{HBV} / \mathrm{Ba}(\mathrm{n}=260)$ than in HBV/C $(\mathrm{n}=197)$ patients. HBV DNA load and HBV genotype were independent factors associated with serum HBsAg levels. Grades of intrahepatic HBsAg expression in $\mathrm{HBV} / \mathrm{C}$ patients were higher than in $\mathrm{HBV} / \mathrm{Ba}$. $\mathrm{HBV} / \mathrm{C}$ transfection led to higher percentage of synthesized HBsAg detained in hepatocytes compared to $\mathrm{HBV} / \mathrm{Ba}$ (100\% vs. $33.0 \%, \mathrm{p}=0.010$ ). Prevalence of large surface gene deletion variants were comparable between two genotypes, also comparable between patients with high and low grades of intrahepatic HBsAg stain. Compared to HBV/B, HBV/C infection led to lower serum HBsAg level but higher intrahepatic HBsAg expression possibly through changing the procedure of HBsAg transport from hepatocytes.

\section{Concurrent Session 7: Fatty Liver Disease 16 Feb, 2009 (Monday), 08:45 - 10:15 Convention Hall A}

Non-alcoholic Fatty Liver Disease in the Asia-Pacific Region

Jian-Gao Fan

Xinhua Hospital, Shanghai Jiaotong University School of Medicine, Shanghai, China

Non-alcoholic fatty liver disease (NAFLD) is the most common liver disorder in Western industrialized countries, affecting $20-40 \%$ of the general population. Based particularly on large community-based studies using ultrasonography, case-control series and prospective longitudinal studies, the prevalence of NAFLD in Asia is between $12 \%$ and $24 \%$, depending on age, gender, locality and ethnicity. There is strong evidence that the prevalence of NAFLD has increased recently in parallel with regional trends in obesity, type 2 diabetes, and metabolic syndrome (MetS); and that further increases are likely. The relationship between NAFLD, central obesity, diabetes, and MetS is clearly evident in retrospective and prospective Asian studies, but the strength of association with these metabolic risk factors is only appreciated when regional definitions of ethnic-specific definitions of central obesity, obesity and MetS are used. Moreover, NAFLD patients with normal body weight seem to more common in Asians than Caucasians. NAFLD appears to be associated with long-standing insulin resistance and likely represents the hepatic manifestation of MetS. Not surprisingly therefore, Asians with NAFLD are at high risk of developing diabetes and cardiovascular disease. In addition, a perspective of NAFLD beyond its hepatic consequences is now warranted; this needs to be considered in relation to management guidelines for affected individuals. Conversely, MetS components may precede the diagnosis of NAFLD. The increasing 
prevalence of obesity, coupled with diabetes, dyslipidemia, hypertension and ultimately MetS puts more than half the world's population at risk of developing NAFLD in the coming decades. Therefore, NAFLD seems to be a major public health concern in the Asia-Pacific Region. In many countries where some estimation of NAFLD prevalence has been made, the magnitude of the problem is comparable to Western countries. Public health initiatives are clearly imperative to halt or reverse the global 'diabesity' pandemic, the underlying basis of NAFLD and MetS.

Recently, NAFLD is also emerging into a new and major health problem in once lean Chinese, where traditionally clinicians have dealt with the formidable burden of chronic hepatitis B. Fatty liver is highly prevalent in China and is more often linked to obesity than to alcoholism. Among more affluent regions of China, the community prevalence of NAFLD is $\sim 15 \%$. With the increasing pandemic of obesity, the prevalence of NAFLD has approximately doubled in the past decade. The full range of histological manifestations of NAFLD has been demonstrated in Chinese patients, but to date hepatic severity is generally mild. In contrast to chronic hepatitis C, steatosis is less common in patients with chronic hepatitis B infection; it is associated with metabolic factors not viral ones, and does not appear to affect disease severity of hepatitis B. Although long-term outcomes of NAFLD in Chinese populations remain unclear, it may be a predictor of metabolic disorders, diabetes and cardiovascular disease. NAFLD should be recognized as part of the MetS and managed in a multidisciplinary approach that addresses liver disease in the context of risk factors for diabetes and premature cardiovascular disease.

\section{Assessment of Nonalcoholic Fatty Liver Disease \\ Vincent W.S. Wong \\ The Chinese University of Hong Kong, Hong Kong SAR}

Nonalcoholic fatty liver disease (NAFLD) is one of the most common chronic liver diseases worldwide. While patients with simple steatosis have excellent long-term prognosis, those with nonalcoholic steatohepatitis (NASH) may progress to cirrhosis, liver failure and hepatocellular carcinoma. NAFLD is also closely associated with metabolic syndrome and cardiovascular events. Therefore, the assessment of NAFLD should focus on (1) establishing the diagnosis and ruling out other causes of abnormal liver function tests, (2) identifying patients who have developed or will develop severe liver injury, and (3) detecting important co-morbid illnesses.

The diagnosis of NAFLD is usually straightforward. By ultrasonographic examination, typical features include bright hepatic echotexture (compared with kidney and/or spleen), deep attenuation and vascular blunting. However, chronic hepatitis $\mathrm{C}$ can cause hepatic steatosis, and a patient with fatty liver can have alternative causes of abnormal liver function tests. Therefore, the minimal diagnostic workup should include hepatitis $\mathrm{B}$ and $\mathrm{C}$ serology and anti-nuclear antibody.

Patients with NASH are more likely to have type 2 diabetes and obesity. Thrombocytopenia, hypoalbuminemia and signs of portal hypertension may be present in patients with NASH-related cirrhosis. However, none of these features are sensitive for NASH or liver fibrosis. Similarly, although serum alanine aminotransferase is commonly used to monitor patients with chronic liver diseases, it has poor correlation with the severity of NAFLD.

At present, the gold standard for the diagnosis of NASH and assessment of liver fibrosis is liver biopsy. However, the procedure carries a small risk of complications, is expensive and may not be acceptable to many patients. Besides, the problem of sampling error is increasingly recognized. Non-invasive tests for NASH and liver fibrosis are urgently needed. Using six clinical parameters (age, hyperglycemia, body mass index, platelet count, albumin, and aspartate aminotransferase-to-alanine aminotransferase ratio), the NAFLD fibrosis score has good negative predictive value for advanced fibrosis in both Caucasians and Chinese. Transient elastography by Fibroscan is another promising non-invasive test for liver fibrosis. It appears to have good accuracy in NAFLD, but failed measurement is more common in obese subjects.

For the metabolic assessment, anthropometric measurements include body weight, body height, waist circumference and hip circumference. Since Asians have the same percentage of body fat and develop metabolic complications at a lower body mass index than Caucasians, it is important to use ethnic-specific cutoff values in anthropometric measurements. Other essential tests include blood pressure measurement, fasting glucose, and fasting lipids. Impaired glucose tolerance and undiagnosed diabetes are very common among NAFLD patients. Oral glucose tolerance test should be considered in every NAFLD patient, especially if the fasting glucose exceeds $5.6 \mathrm{mmol} / \mathrm{L}$

Identification of a Model of Alcohol Preference and its Similarity to Human Alcoholism

Howard C. Thomas

Imperial College at St Mary's Hospital, London

Studies in humans and animals provide evidence that alcohol addiction is a complex disease with both genetic and environmental influences. Mouse models carrying single gene mutations which affect alcohol consumption provide a unique opportunity to approach the molecular complexities underlying the genetic predisposition to drink alcohol.

Using a phenotype-driven ethyl-nitrosourea mutagenesis approach on an alcohol avoiding Balb/c x $\mathrm{C} 3 \mathrm{H}$ background, we have developed a mouse model with strong heritable preference for alcohol consumption. Male heterozygous mutant mice averaged $81.4 \%$ of daily fluid consumption as $10 \%$ ethanol solution $(95 \% \mathrm{CI}=76.4-86.3)$ compared to $9.2 \%(5.3-13.1 \%)$ for wild-type littermates, $\mathrm{p}<0.0001$. Mutant mice consumed significantly greater quantities of alcohol than their wild-type littermates (factor 4.6, 95\% $\mathrm{CI}=3.1-6.2$ ), equating to an average daily intake of $11 \mathrm{~g} / \mathrm{kg}$ bodyweight.

The phenotype is caused by a single base pair mutation producing an L285R amino acid substitution in the third transmembrane domain of the $\mathrm{GABA}_{\mathrm{A}}$ receptor $\beta 1$ subunit. In vitro electrophysiological studies demonstrate spontaneous channel opening in the absence of GABA and a significantly reduced maximal response. In vivo $\mathrm{Mn}^{2+}$ functional MR studies demonstrate increased brain activation in the nucleus accumbens, an area involved in mediating alcohol reward.

We have subsequently tested the role of variation in the Gabrbl gene in determining alcohol dependence in a case control genetic association study in 600 cases of the alcohol dependence syndrome and 500 controls. We found statistically significant evidence of allelic and haplotypic association between three SNPs at the GABAB1 gene and alcoholism.

This mouse model is the first to demonstrate that $\mathrm{GABA}_{\mathrm{A}}$ receptors containing the $\beta 1$ subunit regulate alcohol intake. Translational studies support a role of these receptors in human alcohol seeking behaviour.

Insulin Resistance and Chronic Hepatitis C Virus Infection Jacob George

Westmead Millennium Institute, University of Sydney, Australia

Epidemiological, clinical and laboratory research over the last decade has shown that apart from causing chronic liver injury, hepatitis $\mathrm{C}$ virus infection is associated with extra-hepatic diseases. Amongst these, the metabolic complications of chronic hepatitis $\mathrm{C}$ (CHC) have been the focus of recent study. Large scale prospective reports indicate that $\mathrm{CHC}$ infection is associated with an increased prevalence of type 2 diabetes (T2DM). Smaller scale cross-sectional cohort studies demonstrate an increased prevalence of T2DM in cirrhotic patients with CHC and as well, an increased incidence of T2DM post liver transplantation. This association of $\mathrm{CHC}$ with T2DM appears to be a consequence of elevated insulin resistance in these patients compared to healthy controls and those with other liver diseases. Furthermore, insulin resistance in $\mathrm{CHC}$ is associated with advanced hepatic fibrosis and with more rapid disease progression. Consistent with a causative role, successful antiviral therapy is associated with reductions in insulin resistance. Recently, insulin resistance and T2DM in CHC has been shown to be associated with an elevevated risk for the development of hepatocellular carcinoma. Likewise, insulin resistance adversely impacts on treatment responses (sustained virological response) in patients with genotype 1 and in those with genotype 2 and 3 infections.

The interaction of obesity, hepatic steatosis and insulin resistance with 
disease progression and treatment response has been the subject of debate. Overall, while a role for steatosis and obesity in the milieu cannot be excluded, the weight of evidence suggests that insulin resistance rather than $\mathrm{BMI}$ or steatosis is the principal driver of disease progression and lower treatment-associated viral eradication rates.

The mechanisms for the development of insulin resistance in $\mathrm{CHC}$ are not completely understood. Transgenic mice expressing the HCV core protein develop insulin resistance and hepatic steatosis suggesting a role for at least this protein to the metabolic complications of $\mathrm{CHC}$. In the transgenic mice, TNF production is a major driver for the development of insulin resistance as treatment with TNF antibodies is associated with reversal of the insulin resistance phenotype. In patients with $\mathrm{CHC}$ however, we have been unable to demonstrate a virus specific effect of circulating TNF or a range or other cytokines (IL6, adiponectin and leptin) to the development of insulin resistance. Whether intra-hepatic cytokine levels modulate this metabolic phenotype is at present unresolved. At present, based on in vitro studies, the most plausible explanation for the development of insulin resistance in $\mathrm{CHC}$ appears to be molecular interactions between $\mathrm{HCV}$ proteins such as core and the insulin signalling pathway in hepatocytes.

\section{Concurrent Session 8: HCC Virology \\ 16 Feb, 2009 (Monday), 08:45 - 10:15}

Convention Hall B

\begin{tabular}{l}
\hline HBV Genotypes and HCC \\
Jia-Horng Kao \\
National Taiwan University College of Medicine and National Taiwan \\
University Hospital, Taipei, Taiwan
\end{tabular}

Hepatitis B virus (HBV) is a global health problem. Worldwide, approximately 400 million people are chronic carriers of HBV. HBV infection causes significant morbidity and mortality, ranging from acute or fulminant hepatitis to end-stage liver disease and hepatocellular carcinoma (HCC). HCC is the fifth most common cancer in men and eighth in women in the world, with an estimated 0.5 to 1 million new cases per year and $80 \%$ of them occurring in developing countries including the Asian Pacific region. Overall, $75 \%$ to $80 \%$ of $\mathrm{HCC}$ are attributable to persistent viral infections with either $\mathrm{HBV}(50 \%$ to $55 \%)$ or hepatitis $\mathrm{C}$ virus $(\mathrm{HCV})(25 \%$ to $30 \%$ ). In $\mathrm{HBV}$ endemic countries, chronic $\mathrm{HBV}$ infection has the strongest association with the development of HCC. Several hepatitis B viral factors predictive of clinical outcomes have been identified in the past decade. Among these viral factors, HBV viral load, HBV genotype and subgenotype are worthy of attention.

According to the heterogeneity of viral sequences, at least $8 \mathrm{HBV}$ genotypes (A to $\mathrm{H}$ ) are defined by the divergence in entire HBV genomic sequence of greater than $8 \%$. Epidemiologic studies showed that each genotype has its distinct geographic and ethnic distribution. In addition, HBV genotypes can be further subdivided into subgenotypes by at least $4 \%$ difference in entire genome sequence. Except for genotype E and G, all genotypes have subgenotypes. Epidemiologic data indicate that in genotypes A, B and C, respective subgenotype A1 (Aa) / A2 (Ae), B1 (Bj) / $\mathrm{B} 2(\mathrm{Ba})$ and $\mathrm{C} 1(\mathrm{Cs}) / \mathrm{C} 2(\mathrm{Ce})$ differ widely in many virological aspects.

Ample clinical evidence has recognized that HBV genotypes influence the natural course of liver disease, especially in Asian countries where genotype $\mathrm{B}$ and $\mathrm{C}$ prevail. For example, genotype $\mathrm{B}$ has been shown to be associated with less progressive liver disease than genotype $\mathrm{C}$, and genotype $\mathrm{D}$ has been shown to have a less favorable prognosis than genotype $\mathrm{A}$. Nevertheless, it should be emphasized that all HBV genotype can lead to end-stage liver disease and HCC. In contrast, the clinical relevance of $\mathrm{HBV}$ subgenotypes remains limited. It is suggested that subgenotype $\mathrm{B} 1 / \mathrm{Bj}$ and $\mathrm{C} 1 / \mathrm{Cs}$ may run a more indolent course than $\mathrm{B} 2 / \mathrm{Ba}$ and $\mathrm{C} 2 / \mathrm{Ce}$, respectively. However, further studies are needed to examine the clinical impact of each HBV subgenotype on the progression of liver diseases.

In the meantime, several case-control and prospective studies revealed that the presence of naturally occurring BCP A1762T/G1764A mutation and pre-S deletion are associated with progressive liver diseases. Of particular note is that combination of mutations rather than single mutation in HBV genome was associated with the development of HCC.

In conclusion, on the basis of emerging data, it is recommended that HBV carriers should be routinely genotyped to help identify those who may be at higher risk of liver disease progression and HCC development. In the future, more investigations are needed to clarify the mechanisms involved in HBV genotype-related pathogenesis.

HCC Associated HBV Mutants

Masashi Mizokami

Nagoya City University, Graduate School of Medical Sciences

\section{HBx and HCC}

Irene O.L. Ng

The University of Hong Kong, Hong Kong SAR

Hepatitis B virus (HBV) infection is a major cause of liver cancer (hepatocellular carcinoma, HCC) in Southeast Asia and Hong Kong. HCC is a major malignancy in the world and is particularly prevalent in this region, being the second commonest fatal cancer in Southeast Asia including Hong Kong. Of all the 530,000 HCC cases annually worldwide, $60 \%$ are associated with hepatitis B viral (HBV) infection. In this locality, an even higher portion $(80-90 \%)$ of the HCC is due to chronic HBV infection. Among the four proteins that are encoded from the HBV genome, HBV X $(\mathrm{HBx})$ is the most potentially oncogenic factor. Despite previous extensive studies on the roles of HBx in the initiation of HCC, the roles of HBx in HCC are not well understood. HBx is a transcriptional activator that promiscuously activates several cellular transcription factors including $\mathrm{NF}-\mathrm{kB}, \mathrm{CREB}, \mathrm{AP}-1$ and androgen receptor. $\mathrm{HBx}$ is overexpressed in human HCC. In addition, C-terminal truncation of $\mathrm{HBx}$ has also been frequently found in HCC as a result of HBV integration into cellular genome. Notably, the truncated $\mathrm{HBx}$ mutants derived from $\mathrm{HCC}$ retain some of regulatory activities. Moreover, the truncated $\mathrm{HBx}$ could effectively transform immortalized liver cell line and might contribute to hepatocarcinogenesis. RNA interference (RNAi) against $\mathrm{HBx}$ in $\mathrm{HCC}$ cells which constitutively produce $\mathrm{HBx}$ demonstrated significant reduction in cell growth, anchorage-independent growth in soft agar, and tumor development in nude mice. These findings suggest that $\mathrm{HBx}$ plays an important role in tumorigenicity mechanisms in HCC. Further delineation of deregulation of target genes and signaling pathways by HBx and natural HBx mutants in HCCs will further enhance the understanding how $\mathrm{HBx}$ deregulates important cell pathways and mitotic checkpoint control and exerts tumorigenicity in HCC. Delineation of the mechanism of $\mathrm{HBx}$ in oncogenesis may help design new drugs against HBX or its targets.

\section{Hepatitis C and Hepatocellular Carcinoma \\ T. Jake Liang \\ Liver Diseases Branch, NIDDK, NIH, USA}

Hepatitis C virus infection is a principal etiological agent of liver cancer in the world. The mechanisms of HCV-related oncogenesis remain largely elusive, mostly because of the lack of convenient and suitable tissue culture and animal models and the long duration from infection to cancer development. The pathogenesis of HCV-associated HCC can be viewed in two ways: viral factors and host related events.

Several HCV proteins including core, NS3 and NS5A have been associated with diverse pleiotropic functions including transcriptional activation, signal transduction, in vitro transformation that could be linked to cancer development. Core and NS5A have also been shown to induce the production of reactive oxygen species (ROS) in tissue culture and transgenic animal model. The production of ROS can predispose hepatocytes to DNA damage, which could lead to cumulative mutational events resulting in malignant transformation. 
Other than virus-specific mechanisms that may contribute to carcinogenesis, the chronic "injury and regeneration" model is closely linked to HCC development. Chronic inflammatory changes render a highly carcinogenic environment for the hepatocytes. The production of inflammatory cytokines with generation of reactive oxygen species can induce chromosomal mutations. In addition, the liver is the major organ of detoxifying xenobiotics and toxic by-products. The condition of chronic hepatitis can result in aberrant processing and accumulation of these compounds that are often potent DNA mutagens. As a large number of infected hepatocytes die from host immune response, new hepatocytes are generated and the liver becomes an actively dividing organ. The proliferating hepatocytes, in a mutagenic environment, accumulate mutations and eventually become transformed.

As we understand more about the biology and pathogenesis of the $\mathrm{HCV}$-associated HCC, we may garner valuable information to develop better diagnostic, preventive and therapeutic means.

\section{Concurrent Session 9: Liver Transplantation}

16 Feb, 2009 (Monday), 08:45 - 10:15

\section{Convention Hall C}

Management of HCV in Liver Transplantation
W. Ray Kim
Mayo Clinic College of Medicine, Rochester, MN, USA

Following liver transplantation (LTx), re-infection of the graft with hepatitis $\mathrm{C}$ virus (HCV) occurs almost universally. It is eventually followed by histologic evidence of hepatitis in the majority of patients. Overall, recurrent hepatitis $\mathrm{C}$ in a transplanted liver progresses more rapidly than that in the native liver. Cirrhosis of the transplanted liver may occur in about a third of patients with recurrent hepatitis $\mathrm{C}$ within 5 years of LTx.

In particular, recurrent hepatitis occurring early after LTx has a poor prognosis.

Several factors associated with severe recurrent hepatitis $\mathrm{C}$ have been identified. Older donor age and administration of OKT-3 or bolus doses of corticosteroids are strongest predictors of recurrent hepatitis C. In addition, viral load prior to LTx, CMV infection, tacrolimus (compared to cyclosporine) and steatosis of the donor liver have been proposed as predictors of recurrent hepatitis C. Initially, live donor LTx was postulated to have adverse impact on recurrent hepatitis C. More recent data have largely dispelled such an association.

Because recurrent disease, once it is established, leads to poor outcome, efforts for prevention are warranted. First, in carefully selected patients, antiviral therapy prior to LTx may achieve undetectable levels of HCV RNA. When the so-called low-accelerating dose regimen (LADR) is used, up to $80 \%$ of patients that are RNA-negative at the time of LTx may avoid reinfection of the graft. Second, if possible, older donors are best avoided for recipients with HCV. Third, in the early post-LTx phase, careful considerations in diagnosing and treating acute cellular rejection are necessary. At Mayo Clinic, steroid boluses are reserved only for patients with rejection of moderate degree or worse. Although it is at times difficult to differentiate acute rejection from recurrent hepatitis $\mathrm{C}$, most experienced pathologists are able to diagnose acute rejections that require therapy. In most cases with mild acute cellular rejection, adjustment of maintenance immunosuppression may be sufficient. In contrast to intravenous boluses, avoidance or early withdrawal of oral corticosteroids as a part of maintenance immunosuppression has not been shown to be beneficial. Finally, some have advocated using antiviral therapy in a 'pre-emptive' fashion. In this approach, antivirals (pegylated interferon and ribavirin) are given to patients in the early post-LTx period before recurrent hepatitis $\mathrm{C}$ is established. To date, there is no evidence that pre-emptive antiviral therapy improves patient outcome.

Antiviral therapy for established recurrent hepatitis $\mathrm{C}$ consists of pegylated interferon and ribavirin. 'Protocol' biopsies (biopsies performed at preset interval) are frequently utilized in the decision to initiate therapy. Fibrosis of stage 2 (of 4) or higher is the most commonly used parameter in initiating therapy. However, treatment of recurrent hepatitis $\mathrm{C}$ is fraught with frequent needs for dose reductions and discontinuations. LTx recipients are particularly prone to adverse effects of these medications, most commonly hematologic toxicities. Use of growth factors such as erythropoietin or granulocyte colony stimulating factor is common in this setting. In addition, because of the high prevalence of renal insufficiency among LTx recipients, dose adjustment may be necessary for ribavirin. Sustained viral response (SVR) may be achieved in approximately $25 \%$ of patients. Whether the stopping rules used in non-LTx patients also apply in post-LTx patients is not well-studied, but they are often followed. In patients who fail to achieve SVR, suppressive therapy with reduced doses of pegylated interferon alone has been used, although data in its support are lacking.

In patients whose recurrent hepatitis leads to graft cirrhosis and hepatic decompensation, re-transplantation may be considered. As outcome after re-LTx has been shown to be inferior to that after primary LTx, re-LTx candidates are selected carefully.

Finally, the most serious type of recurrent hepatitis C presents with severe cholestasis (cholestatic hepatitis C), usually early after LTx. Characteristically, these patients have extremely high viral loads. Patients may experience hepatic decompensation and display signs of portal hypertension without clear histologic evidence of cirrhosis. Although some of these patients may response to antiviral therapy, prognosis in general is poor. Re-transplantation in this setting is highly controversial.

Management of HBV in Liver Transplantation

Geoffrey W. McCaughan

Royal Prince Alfred Hospital, Australia

Management of Post-transplant Complications

Chung-Mau Lo

The University of Hong Kong, Hong Kong SAR

Concurrent Session 10: Immunology in Viral Hepatitis

16 Feb, 2009 (Monday), 12:30 - 14:00

Convention Hall A

Update on HBV Vaccine

Mei-Hwei Chang

National Taiwan University Hospital, Taipei, Taiwan

Hepatitis B virus (HBV) infection is highly prevalent in Asia, Africa, Southern Europe, and Latin America. Although complications of chronic HBV infection, such as liver cirrhosis or hepatocellular carcinoma (HCC), develop in later life, primary infection of HBV occurs mainly during infancy or childhood in endemic areas. Universal HBV vaccination program during infancy has effectively reduced the total and chronic infection rates of HBV in the vaccinees. World wide, universal hepatitis B vaccination program has effectively reduced the HBsAg seropositive rates to around one-tenth of the original rates before the programs. Approximately 64 to $75 \%$ of the incidence of HCC in children has been prevented by the universal HBV vaccination program. The mortality rate of fulminant hepatitis in children has also been reduced remarkably after the vaccination program.

Vaccine failure, no vaccination program, and poor compliance are the main problems hindering the success of HBV prevention by vaccination in the world. The causes of vaccine failure includes high maternal viral load, intrauterine infection, vaccine escape mutants, and genetic hyporesponsiveness. Failure to block mother- to infant transmission of HBV is the main cause of vaccine failure. Hepatitis B surface gene mutants in HBV DNA positive children increased gradually from $7.8 \%$ before the vaccination program, to $19.6 \%, 28.1 \%$, and $23.1 \%$ at $5,10,15$ years after the vaccination program. Further works have to be conducted to solve the 
problems.

Long term data after universal hepatitis B vaccination in Taiwan has confirmed that universal HBV vaccination in infancy has produced adequate protection up to 20 years of age. No increase of the chronic HBV infection rate of the vaccinees has been observed up to 20 years old. The new HBV infection rate was not different in those who did and those who did not receive a booster dose of HBV vaccine. Universal booster dose of HBV vaccine is not recommended in vaccinated children and young people under 20 years of age.

\section{HBV-specific CTLs Substantially Influence Outcome of HBV Infection}

\section{in Humans}

Fusheng Wang

Beijing 302 Hospital, Beijing, China

HBV-specific CD8 T-cell responses are thought to be of considerable importance in viral control and immune-mediated liver damage. During acute HBV infection, virus-specific CD8 T-cell responses are often readily detectable and are multi-specific; while these responses are generally weak, and can become exhausted during chronic HBV infection (CHB). HBV-specific CD8 T cells may directly kill virus-infected hepatocytes and contribute to liver pathology. Persistent carriers of HBV, however, fail to generate sufficient cellular immunity against the virus.

HBV-specific T-cell hyporesponsiveness in CHB patients may be involved in the partial, server, full exhaustion or even clone deletion of HBV-specific CD8 T cells. The cellular and molecular mechanisms responsible for the HBV-specific T-cell hyporesponsiveness are probably due to the following factors including negative selection, immunological ignorance, peripheral anergy, dysregulation of lymphokine production and persistent exposure to high viral Ags in hosts. In addition, dendritic cell dysfunction, increased regulatory $\mathrm{T}$ cells and inhibitory $\mathrm{PD}-1 / \mathrm{PD}-\mathrm{L} 1$ pathway in chronic HBV infection has been proposed to impair viral specific CTLs.

Increased regulatory $\mathrm{T}$ cells may contribute to viral persistence in $\mathrm{CHB}$ patients

We found that the circulating Treg frequency was significantly increased in CSHB patients. Treg frequency was positively associated with serum HBV DNA load in CHB patients, and in AHB patients, the Treg frequency was initially at relatively low levels, then increased at the convalescent phase and restored to normal levels upon resolution. Treg were also found to be capable of inhibiting HBV-specific T cell responses in infected patients. Furthermore, we also observed the accumulation of Treg infiltrating in the liver of the CSHB and CHB patients. The results suggest that Treg may play an active role not only in modulating the effectors of the cellular immune response to $\mathrm{HBV}$ infection in peripheral blood as well as in the liver, but also can influence the disease progression of hepatitis B in humans. Current findings suggest that the level of Treg could be a potential prognostic factor and the liver may be a critical site for the specific inhibition of immune response by these cells.

B7-H1 upregulation on myeloid dendritic cells significantly suppresses T-cell immune function

It has been demonstrated that B7-H1 expression is significantly upregulated on circulating $\mathrm{mDCs}$ of $\mathrm{CHB}$ patients compared to healthy individuals, which is significantly correlated with an elevation of serum alanine aminotransaminase levels and plasma viral load. In addition, elevated B7-H1 expression is also closely associated with suppression of T-cell immune function. In vitro blockade of B7-H1 signaling could not only down-regulate interleukin (IL)-10, up-regulate IL-12 production by mDCs but also enhance $\mathrm{mDC}$-mediated allostimulatory capacity and cytokine production of $\mathrm{T}$ cells. These new findings suggested that chronic inflammation may contribute to B7-H1 upregulation on mDCs in CHB patients, which potentially cause defective HBV-specific T-cell function and viral persistence.

Dynamic PD-1 expression on virus-specific CD8 T cells correlates with the outcome of acute hepatitis $\mathrm{B}$ virus infection

We found that PD-1 expression on HBV-specific CD8 $\mathrm{T}$ cells was significantly up-regulated in the early phase of acute HBV infection, and successful viral clearance correlated with a subsequent decrease in PD-1 expression. Blocking PD-1-mediated pathway in vitro, enhanced
HBV-specific CD8 T-cell proliferation and inflammatory cytokine production, but reduced interleukin-10 production and apoptosis, confirming the essential role of PD-1 in tempering T-cell response in the acute phase of infection. In contrast, delayed PD-1 expression on HBV-specific CD8 T cells was associated with acute liver failure. PD-1 up-regulation may efficiently temper pathogenic CD8 T-cell responses and liver damage, correlating with disease progression of acute HBV infection. This study, therefore, reveals new insights into a negative signaling pathway in such early HBV infection, which will be important in better clinical management, prognosis and new treatment.

\author{
Innate Immune Response in $\mathbf{H B V}$ \\ Stephen A. Locarnini \\ WHO Collaborating Centre for Virus Reference and Research, Melbourne, \\ Victoria, Australia
}

Viruses have developed a number of strategies to subvert innate cellular responses, ensuring the survival and persistence of that particular virus in the host. Not surprisingly, the hepatitis B virus (HBV) has also developed a number of strategies to ensure its persistence in the infected host, including the production of excess hepatitis B surface antigen (HBsAg) and the expression of hepatitis $\mathrm{Be}$ antigen $(\mathrm{HBeAg})$. The liver damage of chronic hepatitis $\mathrm{B}(\mathrm{CHB})$ is the result of the host's cellular immune response to HBV-infected hepatocytes as part of the "immune clearance" phase of the disease. Both the innate and adaptive branches of the host immune response need to be considered as a coordinated and dynamic line of attack, rather than as consecutive and independent entities. Both established and potential roles for many components of the innate immune system have been identified. In particular, important interactions between $\mathrm{HBeAg}, \mathrm{HBV}$ and Toll-like receptors (TLR-2), Kupffer cells, natural killer T-cells, and dendritic cells have been described (see Visvanathan, K. et al 2007. Hepatology;45:102). Achieving clearance of HBV appears to require initial viral suppression and recruitment of effector cells by the innate immune system, along with adequate antigen presentation to and activation of the adaptive immune response arm.

\section{Characteristics of Hepatitis B Virus Quasispecies and its Clinical Significances \\ Xinxin Zhang \\ Ruijin Hospital, Jiaotong University School of Medicine, Shanghai, China}

Background/Aims: To investigate dynamic changes of hepatitis B virus (HBV) quasispecies within reverse transcriptase (RT) region in early stage during lamivudine treatment and its prediction for antiviral efficacy. Methods: 21 chronic hepatitis B patients received lamivudine treatment for 48 weeks. 11 patients responded to lamivudine, while 10 patients were nonresponders. HBVDNA from serum samples at baseline and week 4 were extracted. RT region of HBV was amplified, and then cloned and sequenced. Quasispecies complexity and diversity within RT region at baseline and week 4 were analyzed, and viral nucleotide substitution rates during first 4 weeks were calculated. Results: The quasispecies complexity of responders at week 4 were significantly lower than those of nonresponders $(\mathrm{p}<0.01)$. The mean genetic distance (d) and the number of nonsynonymous substitutions per nonsynonymous site $(\mathrm{dN})$ of responders at baseline were significantly higher than those of nonresponders $(\mathrm{p}<0.05)$. At week 4 , the $d \cdot$ the number of synonymous substitutions per synonymous site $(\mathrm{dS})$ and $\mathrm{dN}$ of responders were significantly lower than those of nonresponders $(p<0.01)$. There quasispecies complexity and dS showed no difference between responders and nonresponders at baseline. Viral nucleotide substitution rate of responders was significantly higher than that of nonresponders $(\mathrm{P}<0.05)$. Conclusions: The dynamic changes of HBV quasispecies within RT region showed distinct patterns between responders and nonresponders during lamivudine treatment in the early stage. The HBV quasispecies drifted rapidly to more simple population with less mutation in responders, whereas 
HBV quasispecies evolved slowly to more complex population which prone to mutation in nonresponders. The quasispecies complexity and diversity at week 4 might act as a predictor for short term antiviral efficacy during lamivudine treatment, of which the mechanism needs to be further investigated.

\section{Concurrent Session 11: HCC Treatment $16 \mathrm{Feb}, 2009$ (Monday), 12:30 - 14:00 Convention Hall B}

Prophylaxis of $\mathrm{HBV}$ Reactivation

Winnie Yeo

The Chinese University of Hong Kong, Hong Kong SAR

\section{Surgical Treatment of $\mathrm{HCC}$}

Paul B. S. Lai

The Chinese University of Hong Kong, Hong Kong SAR

Surgical treatment has always been the first-line treatment for hepatocellular carcinoma (HCC). However, there are a number of different choices and sometimes patients may get confused when they are given rather different treatment options by different clinicians.

For liver surgeons, the choice of treatment depends on the liver function, locations and number of tumours, presence or absence of co-morbidities and if there are extra-hepatic spread of tumour. In patients with acceptable risks for surgery, relatively good liver function and tumours located in a segment or a lobe of the liver, surgical resections by means of partial hepatectomy would be the first choice of treatment. However, surgical resections are not risk-free. Although patients nowadays rarely died of massive bleeding during surgery, mortalities are still possible $(<5 \%)$ because of liver failure and sepsis after surgery. Furthermore, mortalities may be related to recurrence of disease and progression of liver cirrhosis in a longer term after initial curative surgical resections. The overall 5-year survival rate after liver resection had significantly been improved to around $50 \%$ in recent years.

If there is no shortage of liver grafts and if the complications related to immunosuppression are excluded from consideration, liver transplantation would become a very viable treatment option for HCC. In patients with HCC confined to the liver, liver transplantation can treat both HCC as well as the underlying cirrhosis. From currently available data, for patients within the Milan Criteria, the 5-year survival had gone up to around $70 \%$. However, liver graft shortage and healthcare resource implications are the major hurdles for liver transplantation to be more widely used as a standard treatment for HCC.

For unresectable or untransplantable HCC, local ablative therapy through percutaneous, laparoscopic or open means are also effective, particular for those tumours which are fewer in number and smaller in size. Loca ablative therapy can be conducted using different energy platforms including radio-frequency ablation (RFA), microwave ablation (MA) or high intensity focused ultrasound (HIFU). Each of these modalities had its own advantages and limitations. The treatment decision may also be affected by availability of the expertise and the machines. Patient selection is also important in the consideration. Overall, for local diseases that are not resectable, local ablative therapy provides effective palliation and even cure for a smaller proportion of cases.

Systemic chemotherapy and trans-arterial treatments in the form of trans-arterial chemo-embolization (TACE) or trans-arterial injection of lipiodol-ethanol mixture (LEM); are commonly used as palliative treatments in patients presented with unresectable tumours. In selected group of patients, tumours may be down-staged so much so that surgical treatments like liver resections or transplantations may be possible.

\section{Targeted Therapy for HCC}

Massimo Colombo

Fondazione IRCCS Maggiore Hospital, Mangiagalli and Regina Elena and University of Milan, Italy

More than $50 \%$ of patients who present at most Centers with a hepatocellular carcinoma (HCC) have an advanced disease, i.e. multiple tumor nodes or a single greater than $5 \mathrm{~cm}$ tumor with portal vein invasion and symptoms. Until 2006, no effective systemic therapy existed for these patients, probably because HCC retains active drug-metabolizing systems that contribute to an intrinsic resistance to chemotherapeutic drugs, while the use of many anticancer drugs was limited by their intrinsic hepatotoxicity that may exacerbate the underlying liver disease. Since HCC is a complex tumor characterized by the aberrant activation of several signaling cascades, including multiple receptor tyrosine kinase pathways involved in growth and angiogenic signaling pathways, targeted therapies have become an important therapeutic option. In the SHARP study conducted in 602 patients with advanced HCC in Europe and USA, the bisaryl urea Sorafenib, which simultaneously inhibits molecular components of the Raf-MEK-ERK signaling pathway involved in tumor growth and VEGF and PDGR receptors involved in neoangiogenesis, was shown to delay the time of radiological progression by $42 \%$ and cancer-related death by $31 \%$. More recently, the beneficial effects of Sorafenib on patients with advanced HCC was confirmed in a phase III study conducted in Asian patients, enrolling patients with more advanced HCCs compared to the SHARP study. Due to the low incidence of objective responses, the clinical effectiveness of sorafenib has created a new paradigm in cancer therapeutics, different from the paradigm of tumor involution and radiological remission typical of the cytotoxic chemotherapies. Interestingly, the beneficial effects of Sorafenib on HCC compares well with other treatments such as Trostuzimab in breast cancer, Bevacizumab in colorectal cancer or Erlotinib in NSC lung cancer (25-35\%). As these studies opened the era of targeted therapy for HCC, other molecules are being evaluated in phase II studies like Erlotinib alone or in combination with Bevacizumab (monoclonal antibody against VEGF), Lapotinib, an EGFR and Her2 receptor blocker, Rapamycin an mTOR inhibitor and Sunitinib a VEGF and PDGF inhibitor, as potential therapies for patients with advanced HCC. To overcome the complexity of genomic aberrations in HCC, combination therapies likely needs to be implemented, though current phase II studies have provided disappointing results for combination therapies with drugs blocking EGFR, VEGF and PDGF receptors and mTOR signaling. Unfortunately, the implementation of targeted therapies is hampered by costs, since $\mathrm{HCC}$ is prevalent in resources-poor countries where access to these drugs is limited. In resources-rich countries, adjuvant therapy with Sorafenib after resection or local ablation has been advocated to improve the outcome of patients with an early HCC. This hypothesis is being tested in a multinational phase III study. Finally, since novel drugs need to be tested in a common frame, a multidisciplinary panel of EASL and AASLD experts proposed survival and time to recurrence as primary end-points for phase III trials assessing primary and adjuvant therapies, respectively. The paradigm of phase II trials was also changed following the advent of targeted therapies, i.e. discouraging the response rate while endorsing time to progression as a reliable end-point to capture clinical benefits.

\section{Chemoembolisation and Ablation for HCC}

Jordi Bruix

Hospital Clínic, University of Barcelona, Spain

Surveillance of patients with liver cirrhosis aims to detect hepatocellular carcinoma (HCC) at an early stage when effective therapies can be successfully applied and provide a benefit in survival. These options include surgical resection, liver transplantation, percutaneous ablation and chemoembolization. According to the BCLC staging and treatment strategy, surgical resection and transplantation are the first treatments to be considered If these are unfeasible patients are considered for percutaneous ablation and if tumor burden exceeds the ciiteria for successful ablation, patients are considered for chemoembolization. 


\section{Percutaneous ablation}

Destruction of tumor cells can be achieved by the injection of chemical substances (ethanol, acetic acid, boiling saline) or by modifying the temperature (radiofrequency, microwave, laser, cryotherapy). The efficacy of percutaneous ablation is assessed by dynamic CT 1 month after therapy. Absence of contrast uptake within the tumor reflects tumor necrosis, while the persistence of contrast uptake indicates treatment failure. The recurrence rate after ablation is as high as for resection. Some recurrences will occur in the vicinity of the treated nodule and are due to the presence of microscopic satellites not included in the ablation zone.

Radiofrequency is now the first technique as it is highly effective and predictable. Child-Pugh A patients with successful tumor necrosis may achieve a $50 \%$ survival at 5 years.

Chemoembolization

The survival benefits of transarterial embolization alone or combined with chemotherapy have been very controversial until recently. Two trials coming from Hong-Kong and Barcelona reported a significant survival benefit for patients treated with chemoembolization and this was further confirmed by a cumulative meta-analysis. It has to be pointed out that the published studies are rather heterogeneous in terms of treatment schedule - interval between sessions, chemotherapy used - but in all of them treatment is associated with a marked antitumoral affect, delay in tumor progression and even prevention of vascular involvement. All in all, these data reinforce the usefulness of chemoembolization for palliation in patients with HCC. The beneficial effect is restricted to patients with preserved liver function (Child-Pugh A) without cancer-related symptoms and thus, only a minor proportion of individuals (around $10-15 \%$ of the whole HCC population) will be thus optimal candidates for this treatment.

Current research aims to increase the efficacy of treatment, reduce the toxicity related to chemotherapy administration and expand the duration of duration of the response. Since ischemia is in the basis of the treatment, it is expected that antiangiogenic agents (ie sorafenib) have the highest potential for effective combination therapy.

\section{Concurrent Session 12: Liver Cirrhosis and Liver Failure 16 Feb, 2009 (Monday), 15:30 - 17:00 Convention Hall A}

Acute on Chronic Liver Failure

Shiv K. Sarin

G B Pant Hospital, New Delhi, India

\author{
Ascites and SBP \\ Anuchit Chutaputti \\ Phramongkutklao Hospital, Bangkok, Thailand
}

Ascites is a common complication of cirrhosis, and is associated with a significant increased portal pressure. The mortality rate in cirrhotic patients hospitalized with ascites is approximately $40 \%$ at 2 years. In mild or moderate uncomplicated cases, salt restriction and anti-mineralocorticoids are the main treatment based on the pathophysiology that secondary hyperaldosteronism, a major factor promoting renal sodium retention. Loop diuretics are also frequently used as an adjunctive therapy. In controlled clinical trials, stepwised medical treatment achieves a response rate in up to $90 \%$ of patients without renal failure.

Ascites that is refractory to diuretic therapy requires either repeated large-volume paracentesis combined with plasma volume expansion. For those in whom greater than $5 \mathrm{~L}$ ascites is removed, it is generally recommended albumin supplement at a dose of $8 \mathrm{~g} / \mathrm{L}$ of ascites removed, to prevent post-paracentesis circulatory dysfunction. A synthetic plasma substitute may be used if the volume of ascites removed is less than $5 \mathrm{~L}$. In selected patients, transjugular intrahepatic portosystemic shunt (TIPS) has provided satisfactory control of ascites. TIPS is more effective than paracentesis to prevent recurrence of ascites. Furthermore, the TIPS patients had a significantly lower risk of developing hepatorenal syndrome (HRS). However, the risk of severe hepatic encephalopathy and the cost of therapy were higher in the TIPS group, while the results regarding survival were discrepant according to the different reports. The most recently published meta-analysis found that the actuarial probability of transplant free survival was better in patients allocated to the TIPS group than to the paracentesis group which could result from the reduction of life threatening complications such as spontaneous bacterial peritonitis (SBP), HRS or variceal bleeding. Any patients with cirrhosis and ascites should be considered a potential candidate for liver transplantation, because the long-term prognosis of patients with ascites is poor.

SBP is a bacterial infection of ascitic fluid which arises in the absence of any other source of sepsis within the peritoneums. It is recognized as a common and severe complication in patients with cirrhotic ascites. Numerous studies suggest that $8-27 \%$ of hospitalized patients with cirrhosis and ascites have SBP, with in-hospital mortality ranging from $20-40 \%$. Even in the era of new antibiotics, mortality levels remain significant (10-20\%). Patients with an ascitic fluid polymorphonuclear leucocyte count of greater than $250 / \mathrm{mm}^{3}$ should be considered to have SBP regardless of ascitic fluid culture result. The condition must be diagnosed and treated promptly. Hence, the use of urine reagent strips for diagnosis of SBP by detection of leucocyte esterase activity, by colorimetric method, in the ascitic fluid has been suggested. Recently, a critical review has been performed on 19 publications featuring one or more ascitic fluid samples in cirrhotic patients and a comparison between the reagent strip results and the corresponding cytological and bacteriological analysis of the ascitic fluid. Five strips with different grading scales were studied. The sensitivity varies $(45-100 \%)$ depending on the type of the strip and the cut-off used for diagnosis. However, regarding the low sensitivity and the high risk of false negatives, especially in patients with SBP and low polymorphonuclear count, the reagent strip test cannot be recommended for the diagnosis of SBP.

Empirical antibiotic therapy must be initiated immediately after the diagnosis of the infection is made. Since Gram-negative aerobic bacteria from the family of Enterobacteriaceae and non-enterococcal Streptococcus spp. are the most common causative organisms, the initial empirical antibiotic therapy of SBP should cover these organisms. Cefotaxime has been the most extensively investigated in patients with SBP.

In one third of patients with SBP, renal impairment develops despite treatment of their infection with non-nephrotoxic antibiotics. This deterioration of renal function is the most sensitive predictor of in-hospital mortality. The result of a prospective study shows that the development of bacterial-induced renal failure in patients with cirrhosis and ascites is related to the model for end-stage liver disease (MELD) score, and to both the severity and the lack of resolution of the infection. A progressive form of renal failure occurs as a consequence of biliary or gastrointestinal tract infections, SBP, and urinary tract infection. Treatment with intravenous albumin $(1.5 \mathrm{~g} / \mathrm{kg}$ of body weight on the diagnosis of SBP and $1 \mathrm{~g} / \mathrm{kg}$ after 2 days) in addition to an antibiotic reduces the incidence of renal impairment $(10 \%$ vs. $33 \%)$, in-hospital mortality ( $10 \%$ vs. $29 \%)$, and mortality at 3 months ( $22 \%$ vs. $41 \%)$.

The risk of developing SBP is greater in those with a coexisting gastrointestinal hemorrhage, a previous episode of SBP or low ascitic protein levels. Antibiotic prophylaxis should be administered to cirrhotic patients with gastrointestinal hemorrhage, independently of the presence or absence of ascites to prevent bacterial infection and improve survival. Continuous oral administration of norfloxacin is recommended in patients recovering from an episode of SBP. In cirrhotic patients without a past history of SBP and in whom ascitic fluid protein concentration is less than $10 \mathrm{~g} / \mathrm{L}$, primary antibiotic prophylaxis reduces the incidence of SBP, nevertheless, there is no improvement in survival and increase in number of drug resistance. However, it is justified that antibiotic prophylaxis should be considered in patients with low-protein ascites or bilirubin greater than $2.5 \mathrm{mg} / \mathrm{dL}$ during hospitalization. Cirrhotic patients with more advanced disease have greater risk to develop SBP. A retrospective case-control study in patients with cirrhosis and ascites who underwent diagnostic paracentesis upon hospital admission shows that increasing MELD score is independently associated with a greater risk of SBP. In patients with ascitic fluid protein $<15 \mathrm{~g} / \mathrm{L}$ and one of the following: a) advanced liver failure, b) impaired renal function, c) hyponatremia, 
prophylactic antibiotic should be administered indefinitely to reduce the risk of SBP and HRS and to improve survival. Since the 2-year survival of cirrhosis with SBP is grim, liver transplantation is the only option to improve survival.

Antiviral therapy for hepatitis B virus associated hepatic failure Yuming Wang

Institute for Infectious Diseases of PLA, Southwest Hospital of the Third Military Medical University, Chongqing, China

Background: To reviews the current strategies used in the antiviral treatment of HBV-associated fulminant hepatic failure.

Methods: Literature search was conducted using PubMed on the related subjects. Part of the material was based on the most recent work in the authors' laboratory.

Results: The efficacy of antiviral therapy for HBV-associated hepatic failure hepatitis B has been well demonstrated. Successful and prolonged inhibition of viral replication can prevent recurrent $\mathrm{HBV}$ infection after orthotopic liver transplantation (OLT), delay the development of cirrhosis, liver failure and reducing the risk for primary hepatocellular carcinoma (HCC)(Table 1 and Figure 1). Combination therapies and newer agents such as entecavir, telbivudine, tenofovir and others have become more and more widely used and have potential application in antiviral therapy.

Conclusions: The prognosis of HBV-associated fulminant hepatic failure is being transformed by developments in antiviral therapy, and newer strategies may help to solve the current problems.

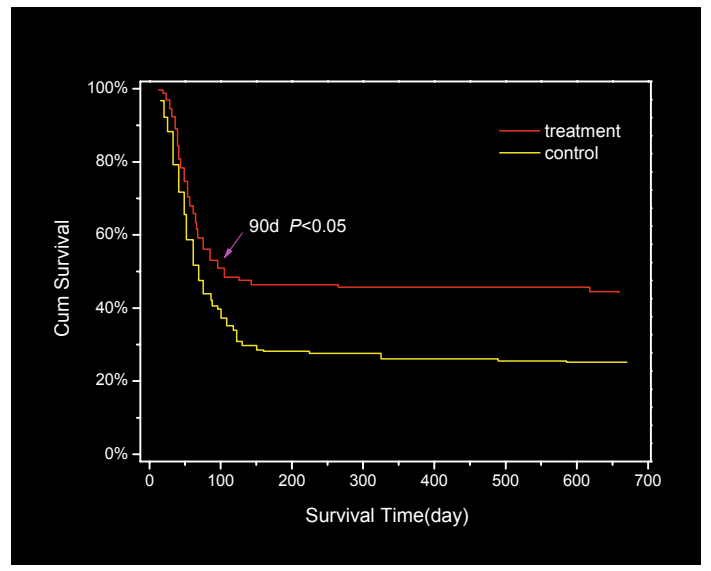

Fig. 1. Survival rates in 215 liver failure patients with hepatitis B after lamivudine treatment.

\begin{tabular}{|c|c|c|c|c|c|}
\hline \multirow{2}{*}{ Year (n) } & \multicolumn{3}{|c|}{ improvement/total (\%) } & \multirow[b]{2}{*}{$\chi^{2}$} & \multirow[b]{2}{*}{$P$} \\
\hline & stage : & early & late & & \\
\hline $2000(19)$ & & $2 / 5(40.0)$ & $2 / 14(14.3)$ & 1.86840 & .1717 \\
\hline $2001(49)$ & & $6 / 15(40.0)$ & $4 / 34(11.8)$ & 5.10480 & .0238 \\
\hline $2002(74)$ & & $12 / 24(50.0)$ & $7 / 50(14.0)$ & 11.01290 & .0009 \\
\hline $2003(100)$ & & $15 / 31(48.4)$ & $16 / 69(23.1)$ & 6.34970 & .0117 \\
\hline 2004 (167) & & 28/52(53.9) & $29 / 115(25.2)$ & 13.05440 & .0003 \\
\hline 2005 (189) & & $31 / 51(56.1)$ & $39 / 132(29.5)$ & ) 5.09750 & .0240 \\
\hline $2006(212)$ & & $40 / 69(58.0)$ & $49 / 143(34.3)$ & ) 4.17040 & .0411 \\
\hline
\end{tabular}

Table 1. Curative effect in 810 liver failure patients of hepatitis B after nucleoside analogue treatment

\section{Management of Decompensated Chronic Hepatitis B \\ Robert J. Fontana \\ University of Michigan Medical School, Ann Arbor, MI, USA}

Chronic hepatitis B virus (HBV) infection is a leading cause of death worldwide through the development of liver failure and/ or hepatocellular carcinoma (HCC). The poor prognosis of untreated patients with decompensated $\mathrm{HBV}$ is, in part, related to the disease severity at presentation (i.e. CTP score, MELD score) as well as the presence of active viral replication (i.e HBV DNA level). A randomized controlled study of chronic HBV patients with compensated cirrhosis demonstrated a clinically significant reduction in disease progression in patients treated with lamivudine compared to placebo. In addition, multiple cohort studies have demonstrated that lamivudine is well tolerated and associated with improved clinical outcomes in patients with decompensated HBV. However, the clinical benefit with the oral nucleos $(\mathrm{t})$ ide analogues often takes 3 to 6 months and HCC may develop despite clinical improvement.

Practice guidelines recommend initiating an oral antiviral agent for decompensated HBV patients independent of their HBeAg status and serum ALT and HBV DNA levels. However since some decompensated patients have a poor short-term prognosis despite antiviral therapy (i.e. high bilirubin), concomitant evaluation for liver transplantation is advisable. Entecavir and tenofovir are considered first line agents in previously untreated decompensated HBV patients due to their rapid suppression of HBV DNA and low rates of drug resistance. However, studies regarding their efficacy and safety in decompensated HBV either alone or in combination with other oral agents are limited. Although lamivudine has been widely used in decompensated HBV, this drug is now considered a $2^{\text {nd }}$ line agent due to the high rate of drug resistance with prolonged dosing and potential for disease flares with viral breakthrough. Telbivudine, a potent suppressor of HBV replication, is also considered a $2^{\text {nd }}$ line agent in decompensated HBV due to the increasing rate of drug resistance with prolonged dosing. Finally, adefovir combined with lamivudine has been widely used in decompensated HBV patients with lamivudine-resistant HBV. However, the slower antiviral effect of adefovir coupled with the increasing rate of drug resistance make adefovir a $2^{\text {nd }}$ line agent for previously untreated patients.

After starting antiviral therapy in decompensated patients, serial HBV DNA levels should be obtained every 3 to 4 months as well as monthly liver biochemistries to assess response. In patients with rising HBV DNA, confirmatory testing for possible drug-resistant HBV should be obtained after insuring compliance. In subjects with worsening liver function despite HBV DNA suppression, evaluation for HCC, portal vein thromboses, or other hepatotoxic insults should be considered. In subjects with lamivudine-resistant decompensated $\mathrm{HBV}$, the nucleotide analogues, tenofovir and adefovir, are preferred over entecavir and telbivudine due to their lack of cross-resistance. Monitoring of creatinine and phosphate levels is recommended when using the nucleotide analogues in decompensated patients as well as increasing the dosing interval in patients with renal impairment.

Overall, the increasing use of antiviral agents for decompensated HBV has been associated with a decline in the listing of decompensated HBV patients for liver transplantation in the United States. Nonetheless, subjects who require transplantation should receive the most effective oral antiviral regimen to suppress HBV DNA to low or undetectable levels and continue on these agents in combination with HBIG post-transplant to minimize the risk of allograft reinfection.

\section{Concurrent Session 13: HCC Basic \\ 16 Feb, 2009 (Monday), 15:30 - 17:00 Convention Hall B}

Differential Expressed $m i R-223$ and $m i R-222$ in the Development of Hepatocellular Carcinoma 
Nathalie Wong

The Chinese University of Hong Kong, Hong Kong SAR

Recent advances in cancer biology have emphasized on alterations of endogenous microRNAs (miRNAs) expressions as an important event in the tumorigenesis of human cancers. MiRNA belongs to a family of small non-coding RNAs ( 18-22nt) that regulates gene expression by either directing mRNA degradation or repressing protein translation. It is also becoming apparent that cancer-associated miRNAs may be regarded as a new class of tumour suppressors and oncogenes capable of regulating vital signalling paths.

Though rare in the West, Hepatocellular Carcinoma (HCC) is prevalent in Southeast Asia including Hong Kong. Extensive genetic analyses have provided valuable information on vital genomic and transcriptional alterations of $\mathrm{HCC}$, but information on differential expressed miRNA remains largely undefined. We undertook microarray profiling for changes of cellular miRNA expressions in a large panel of HCC cell lines. Distinct up-regulations of miR-222, miR-221 and miR-31 and down-regulations of miR-223, miR-126 and miR-122a were suggested. Specific miR-222 and miR-223 deregulations were further verified in an independent series of tumors. By quantitative PCR analysis, we found the highly deregulated miR-222 and miR-223 could unequivocally distinguish HCC tumors from adjacent non-tumoral liver, irrespective of viral etiologies. In functional analysis, re-expression of miR-223 in HBV, HCV and non-B non-C related HCC cell lines revealed a consistent growth inhibitory effect. Integrative analysis of mRNA array with in-silico predictions defined Stathmin1 $(S T M N 1)$ as a potential downstream target of miR-223. A strong inverse relationship between $S T M N 1$ transcriptions and protein levels with miR-223 expressions was indeed suggested. We further showed that miR-223 could readily suppress the luciferase activity in reporter construct containing the STMN1 3'UTR, which in turn confirmed target association. In summary, our study revealed specific miRNA expressions in HCC tumours, and underscores importance on miR-223 and miR-222 in the development of HCC.

\section{Cancer Stem Cells of Hepatocellular Carcinoma}

Ronnie T.P. Poon

The University of Hong Kong, Hong Kong SAR

Hepatocellular carcinoma (HCC) is a tumor associated with rapid growth and high propensity for vascular invasion and metastasis. Recently, evidence form other cancers has supported the hypothesis that a small population of cancer cells with characteristics similar to normal stem cells, called cancer stem cells (CSCs), are responsible for self-renewal and growth of the tumor. Specific surface markers that characterize CSCs in other common human cancers have been identified. However, there is a relative paucity of knowledge on CSCs in HCC.

Using a panel of HCC cell lines, our group has identified CD90 as a marker of CSCs in HCC. The number of CD90+ cells increase with tumourigenicity of HCC cell lines. Our group reported the first successful isolation of CD45-CD90+ CSCs from human HCC specimens. CD45-CD90+ cells were absent in normal, cirrhotic, and parallel nontumorous livers. CD45-CD90+ cells from the tumor tissues of liver cancer patients generated tumor nodules in immunodeficient mice, and serial transplantation of CD90+ cells from tumor xenografts generated further tumor nodules in subsequent batches of immunodeficient mice, demonstrating the self-renewal capacity of these CSCs. In addition, CD45-CD90+ cells were detected in $90 \%$ of blood samples from liver cancer patients, but none in normal subjects or patients with cirrhosis. High level of CD45-CD90+CD44+ CSCs in the circulation predicted a higher recurrence rate after resection of HCC. Treatment of CD90+CD44+ CSCs with anti-human CD44 antibody induced cell apoptosis in a dose-dependent manner. CD90+ CSCs are resistant to chemotherapy, but the use of anti-human CD44 antibody can enhance chemosensitivity of $\mathrm{CD} 90+$ cells. In conclusion, identification of CD45-CD90+ CSCs in both tumor tissues and circulation suggests that CD45-CD90+ could be used as a prognostic marker for human liver cancer, and more importantly, as a target for the therapy to enhance the chance of eradicating this cancer.
Sex Steroid Receptors, the Key for Determining the Gender Disparity of Hepatocellular Carcinoma

Shiou-Hwei Yeh

NTU Center for Genomic Medicine, Taipei, Taiwan

Men have a higher incidence of hepatocellular carcinoma (HCC) than women. The epidemiologic and animal studies suggested that it might due to the stimulatory effects of androgen and protective effects of estrogen, with the molecular mechanisms remained largely unknown. Our recent studies provided two novel mechanisms for explaining the higher androgen activity identified in male HCCs and also the lower estrogen activity identified in female HCCs. First, we have identified that HBx can enhance the transcriptional activity of androgen receptor (AR). The increased carcinogenic effect of $\mathrm{HBx}$ enhanced AR activation was demonstrated by the cell culture based anchorage independent colony forming assay and was also suggested by the HBx transgenic mouse model, in an androgen concentration dependent manner. The results first provide an explanation for the male preference of HBV-related HCCs. We have further identified that $\mathrm{HBx}$-enhanced $\mathrm{AR}$ transactivation is mainly through its enhancing two critical steps of ligand-stimulated AR activities, the dimerization step and the AR N-terminal transactivation domain (NTD) activation step. The key switches for these two events have been further delineated. HBx increases the NTD activation of the AR through c-Src kinase and enhances AR dimerization by inhibiting the glycogen synthase kinase-3 $\beta$ (GSK-3 ) activity, which acts as a negative regulator of the interaction between AR and the $\mathrm{N}$ - and $\mathrm{C}$-termini.

Secondly, in our studying whether there were differences in levels of microRNA (miRNA) molecules between male and female HCC, the expression profiles of a panel of candidate miRNAs were compared between liver samples taken from male and female HCC patients. We identified a miRNA miR-18a to be significantly elevated specifically in female HCCs. The gene ESR1, which encodes the estrogen receptor- (ER ), was identified as a target of miR-18a. This miRNA can repress ER translation by binding to its mRNA at the 3'UTR. Our study thus provides a novel miRNA-mediated regulatory mechanism for controlling the ER expression in hepatocytes. miR-18a prevents translation of ER , potentially blocking the protective effects of estrogen and promoting the development of HCC in women.

Our current studies demonstrated that the gender disparity of HCC is attributed by both androgen and estrogen sex hormone pathways, with distinct roles in each gender. Therefore, the ligand and the receptor factors of both sex hormones need to be included for assessing the relative risk of HCC patients of each gender.

\section{Molecular Markers for Tumor Recurrence after Hepatectomy for Hepatocellular Carcinoma \\ Huichuan Sun \\ Liver Cancer Institute and Zhongshan Hospital, Fudan University, Shanghai, China}

Tumor recurrence remains a serious problem in patients after hepatectomy for hepatocellular carcinoma (HCC), therefore, identifying of patients with a high risk of recurrence is the first step to prevent recurrence. Meanwhile, emerging role of molecular targeting therapy requires the treatment is applied on an individual basis. Molecular markers to predict the risk of recurrence consist of several categories of risk factors, such as patient factors and tumor factors considering the role of active interaction between tumor and host in carcinogenesis and tumor progression or metastasis. To add the complexity, tumor recurrence may come from a real new tumor originated from an irrelevant carcinogenesis, and an occult residual cancer related with the initial one, which is also regarded as the early recurrence (residual cancer) and late recurrence (multicentric cancer) based on the time to recurrence. Molecular markers from tumor tissues or tumor cells still remain the 
mainstay to predict the risk of recurrence based on the conception that the behavior of tumor cell is predominantly determined by its specific genome/gene expressions. A large number of individual genes or gene expression signatures (a set of genes) have been identified to associate with either recurrence or invasiveness and metastasis feature, which is used to be surrogate marker for recurrence.

The recent progress in this field is the role of peri-tumoral liver tissue has been recognized, however, it also needs to discrete its role in predicting the risk of occult residual cancer or de novo carcinogenesis, because the results came from either $\mathrm{HBV}$ or $\mathrm{HCV}$ predominant populations.

So far, the significance of molecular markers for tumor recurrence is still within the lab to help us to understand the mechanisms. When the hype becomes a hope is still a question.

\section{Concurrent Session 14: Pancreatobiliary Disease 16 Feb, 2009 (Monday), 15:30 - 17:00 Convention Hall C}

EUS

Yuk Tong Lee

Hong Kong Baptist Hospital, Hong Kong SAR

Endoscopic ultrasonography (EUS) combines both endoscopic and high frequency ultrasound examination into one. It has revolutionized the clinical management of pancreatic and biliary diseases in recent years.

For pancreatic cancer diseases, it has been shown that EUS is highly sensitive in detecting the presence of pancreatic cancer, especially for small tumour that could not be seen by other imaging techniques. In a retrospective analysis of 117 patients who had undergone CT scan and EUS examination, the sensitivity and accuracy for CT scan in detecting the presence of pancreatic tumour were $93 \%$ and $90 \%$, as compared with $99 \%$ and $91 \%$ for EUS. The major difference was in the detection of small tumour $(\leq 2 \mathrm{~cm})$, in which the sensitivity for EUS was much higher than CT examination $(96 \%$ vs $83 \%$ ). EUS could confirm or reject the suspicion of pancreatic lesion that is incidentally found by other imaging. The use of pre-operative EUS-FNA could avoid inappropriate operation in patients with metastatic disease or autoimmune pancreatitis. For pancreatic cystic lesions, it is difficult to differentiate the nature of the cysts by morphological study only. Adding EUS-FNA for sampling cystic fluid for analysis, the diagnostic accuracy would be increased. Various EUS-guided innovative pancreatic therapies are being explored in recent years, which include EUS guided pancreatic duct drainage, EUS guided local ablation of small tumours, EUS guided injection therapy with different agents, and EUS guided implantation of radioactive substances for localized interstitial radiation therapy for advanced stage pancreatic cancer

Since the bile duct is very close to the duodenum, EUS is shown to be highly accurate in detecting and differentiating causes of biliary obstructive disease including pancreatic and ampullary tumours, and common bile duct (CBD) stone. The accuracy of EUS in detecting CBD stone exceeds $95 \%$. In contrast to MRCP, the accuracy for EUS would not be affected by the size of the stone or the diameter of the bile duct. Therefore, EUS is a valuable tool for triaging patients to receive invasive ERCP investigation.(figure) In a recent prospective randomized study, patients, who were suspected to have biliary obstructive disease, were randomized to receive direct ERCP or EUS guided management protocol. There was no clinical, biochemical or radiological parameters between two groups that were useful in predicting the presence of CBD stones. In EUS group, all the CBD stones and sludge were identified. Compared with the ERCP group, EUS group had less complication and lower mortality rate. More than $75 \%$ of the ERCP procedures could potentially be spared by the EUS guided management approach. In another study, EUS could help to reduce the need of ERCP in patients with acute biliary pancreatitis due to its high sensitivity and negative predictive values in diagnosing CBD stone.

To gain access to the bile duct for endotherapy is sometimes difficult. Various new EUS guided biliary interventions, including EUS guided choledochoduodenostomy and hepaticogastrostomy, are being used in patients with difficult biliary access. It will set a new paradigm for future biliary endoscopists and endosonographers in treating pancreatobiliary diseases.

\section{PBC vs AIH}

Michael P. Manns

Medical School of Hannover, Germany

\author{
Primary Sclerosing Cholangitis and Cholangiocarcinoma \\ Rupert W.L. Leong \\ Concord and Bankstown Hospitals, Sydney Australia
}

Primary sclerosing cholangitis (PSC) is characterised by idiopathic intra- and extra-hepatic biliary ductal inflammation, fibrosis and cholestasis. No medical therapy exists and the condition generally progresses to biliary cirrhosis and liver failure. PSC is most commonly diagnosed in the 30-40's year age group and more commonly in men. There may be epidemiological differences between Western and Asian PSC cohorts. Approximately $75 \%$ of PSC is linked with inflammatory bowel disease especially ulcerative colitis Diagnosis is initially suspected based on cholestatic LFT's. Anti-neutrophil cytoplasmic antibody (ANCA) and other serological markers may be present and histology is non-specific. Cholangiography by ERCP or MRCP is the gold standard in diagnosis and show multi-focal ductal strictures and beading. In a variant known as small-duct PSC, these cholangiographic changes may be absent. Ursodeoxycholic acid, a hydrophilic bile acid, may improve PSC through stimulation of hepatobiliary secretion, inhibition of apoptosis and the protection of bile epithelial cells from the toxic effects of hydrophobic bile acids. ERCP stenting of dominant strictures may be helpful. However, at present, liver transplantation is the only treatment offering reversal of end-stage liver disease and indications include complicated cirrhosis, intractable pruritus, fatigue, and jaundice refractory to endoscopic or medical treatment

A further complication of PSC is cholangiocarcinoma, which is characterised clinically by a poor prognosis. A major diagnostic dilemma remains differentiating early and potentially resectable cholangiocarcinoma from benign strictures in the setting of PSC and other mimics. Cytology brushing with or without combined biopsies has a limited yield but the addition of molecular markers and detection of genetic aberrations may improve diagnostic accuracy. A serum Ca19-9 tumour marker level of $100 \mathrm{U} / \mathrm{ml}$ or greater is reported to have a sensitivity of $75 \%$ and specificity of $80 \%$ in PSC. Transabdominal ultrasound and cross-sectional imaging remain firstline investigations and cholangiography with ERCP, MRCP and PTC are essential for assessing tumour extent and resectability. Advances in peroral cholangioscopy such as the Spyglass Direct Visualization System, may allow directed intra-ductal biopsies of indeterminate strictures. Spyglass is a steerable intraducta fibre-optic system with a $1.2 \mathrm{~mm}$ working channel for biopsies and endotherapy and is operated by a single endoscopist. Other imaging diagnostic techniques include intraductal/ endoscopic ultrasound, PET CT, and virtual biopsy technologies. Surgery is the only curative treatment for cholangiocarcinoma. Liver transplantation following preoperative chemoirradiation in carefully selected patients has been reported. The majority of patients with cholangiocarcinoma are treated palliatively with biliary endoprostheses.

\section{Acute Pancreatitis}

John Wong

The Chinese University of Hong Kong, Hong Kong SAR

Acute inflammation of the pancreas is a common emergency condition, which ranges from very mild and self-limiting, to a fatal situation. The overall reported mortality can be up to $10 \%$. With conservative management, $75 \%$ of patients can recover completely after a few days, but in the remainder, the pancreatitis attack can be fulminant, with a mortality up to 
$40 \%$.

Well known aetiologies of acute pancreatitis include gallstones disease and alcohol consumption. Other predisposing factors including viral infection, trauma, hypercalcaemia, hyperlipidaemia, renal failure and lesions leading to pancreatic duct obstruction have been described. Pathophysiology is still not fully understood. Proposed mechanism included acinar rupture with release of activated enzymes and autolysis within the gland parenchyma. In the severe form, a systemic inflammatory response occurs with release of multiple inflammatory mediators and activation of the cytokine cascade.

Early identification of severe acute pancreatitis is desirable, allowing early intensive monitoring. The Ranson score, modified Glasgow criteria and the APACHE II system have been used for prediction of severity. The role of CT scanning for grading of severity will be discussed, as well as the increasing accuracy of magnetic resonance as a valuable alternative.

Evidence for the efficacy of early ERCP and endoscopic sphincterotomy will be presented. Randomized controlled studies have shown improvement in morbidity and reduction in mortality. Patients with signs of jaundice and/or with cholangitis component need to be identified early. Total parenteral nutrition (TPN) has been employed for over 40 years for patients with severe acute pancreatitis. Recent studies have examined the safety of early enteral feeding, especially through the nasojejunal route.

The place of antibiotic therapy in severe pancreatitis has been widely examined. The use of intravenous imipenem in a dose of $500 \mathrm{mg}$ three times daily has claimed a reduction in the incidence of infected pancreatic necrosis in a multicentre randomized Italian study.

The role of surgery in acute pancreatitis will be addressed. It is strongly recommended that patients having gallstones should have a laparoscopic cholecystectomy performed during the same hospital admission to prevent recurrent attacks. Those older patients who are considered unfit for general anaesthesia may be managed legitimately by endoscopic sphincterotomy. Recommendation for sterile necrosis is to avoid surgery. Pancreatic necrosectomy and drainage is the recommended approach when infection develops. Around $50 \%$ of pancreatic pseudocysts will resolve spontaneously on conservative treatment. Internal drainage by laparoscopic surgery or endoscopic stents have been gaining popularity. 